\title{
Simultaneously vanishing higher derived limits
}

\author{
Jeffrey Bergfalk ${ }^{1,2}$ and Chris Lambie-Hanson ${ }^{\left({ }^{2}\right.} 3$ \\ ${ }^{1}$ Universität Wien, Institut für Mathematik, Kurt Gödel Research Center, Kolingasse 14-16, 1010 Wien, Austria; \\ ${ }^{2}$ Centro de Ciencas Matemáticas, UNAM, A.P. 61-3, Xangari, Morelia, Michoacán, 58089, México; \\ E-mail: jeffrey.bergfalk@univie.ac.at. \\ ${ }^{3}$ Department of Mathematics and Applied Mathematics, Virginia Commonwealth University, Richmond, VA 23284, USA; \\ E-mail: cblambiehanso@vcu.edu.
}

Received: 28 April 2020; Revised: 11 April 2021; Accepted: 17 March 2021

2020 Mathematics Subject Classification: Primary - 03E05; Secondary - 03E75, 03E55, 55N07, 55N40, 18E25

\begin{abstract}
In 1988, Sibe Mardešić and Andrei Prasolov isolated an inverse system A with the property that the additivity of strong homology on any class of spaces which includes the closed subsets of Euclidean space would entail that $\lim ^{n} \mathbf{A}$ (the $n$th derived limit of $\mathbf{A}$ ) vanishes for every $n>0$. Since that time, the question of whether it is consistent with the ZFC axioms that $\lim ^{n} \mathbf{A}=0$ for every $n>0$ has remained open. It remains possible as well that this condition in fact implies that strong homology is additive on the category of metric spaces.

We show that assuming the existence of a weakly compact cardinal, it is indeed consistent with the ZFC axioms that $\lim ^{n} \mathbf{A}=0$ for all $n>0$. We show this via a finite-support iteration of Hechler forcings which is of weakly compact length. More precisely, we show that in any forcing extension by this iteration, a condition equivalent to $\lim ^{n} \mathbf{A}=0$ will hold for each $n>0$. This condition is of interest in its own right; namely, it is the triviality of every coherent $n$-dimensional family of certain specified sorts of partial functions $\mathbb{N}^{2} \rightarrow \mathbb{Z}$ which are indexed in turn by $n$-tuples of functions $f: \mathbb{N} \rightarrow \mathbb{N}$. The triviality and coherence in question here generalise the classical and well-studied case of $n=1$.
\end{abstract}

\section{Introduction}

A main and organising theme in the study of infinitary combinatorics is the phenomenon of incompactness; broadly speaking, the term denotes settings in which the local and global behaviours of a structure sharply diverge. Nontrivial coherent families of functions on a variety of index sets form one prominent class of examples. These connect in turn to local-to-global questions in homology theory, wherein the nontrivial coherence relations of such set-theoretic interest figure as only the first in an infinite family of incompactness principles given by the derived functors $\lim ^{n}$ of the inverse limit of various inverse systems. For one particular inverse system, denoted $\mathbf{A}$ in what follows, the question of whether these associated incompactness principles can simultaneously fail had been longstanding; the main result of this paper is that under the assumption of the existence of a weakly compact cardinal, they can. This result carries implications for the strong homology of metric spaces and, as we note below, possibly for other areas of mathematics as well.

We begin by reviewing the historical background to this result; it traces to Sibe Mardešić and Andrei Prasolov's 1988 paper 'Strong homology is not additive' [22]. Their title references the following potential continuity property of a homology theory:

(C) The Author(s), 2021. Published by Cambridge University Press.. This is an Open Access article, distributed under the terms of the Creative Commons Attribution licence (http://creativecommons.org/licenses/by/4.0/), which permits unrestricted re-use, distribution, and reproduction in any medium, provided the original work is properly cited. 
Definition 1.1 ([23]). A homology theory is additive on the class $\mathcal{C}$ of topological spaces if for every natural number $p$ and every family $\left\{X_{\alpha} \mid \alpha \in A\right\}$ with each $X_{\alpha}$ and $\amalg_{A} X_{\alpha}$ in $\mathcal{C}$, the map

$$
i_{p}: \bigoplus_{A} \mathrm{H}_{p}\left(X_{\alpha}\right) \rightarrow \mathrm{H}_{p}\left(\coprod_{A} X_{\alpha}\right)
$$

induced by the inclusion maps $\iota_{\alpha}: X_{\alpha} \hookrightarrow \bigsqcup_{A} X_{\alpha}$ is an isomorphism.

In [22], Mardešić and Prasolov isolated an inverse system A with the property that the additivity of strong homology on any class of topological spaces which includes the closed subsets of Euclidean space would entail that $\lim ^{n} \mathbf{A}=0$ for all $n>0$. They also succeeded in casting the vanishing of $\lim ^{1} \mathbf{A}$ in the following combinatorial terms: $\lim ^{1} \mathbf{A}=0$ if and only if for every family of functions

$$
\Phi=\left\langle\varphi_{f}:\{(k, m) \mid m \leq f(k)\} \rightarrow \mathbb{Z} \mid f \in{ }^{\omega} \omega\right\rangle
$$

whose elements agree pairwise modulo finite sets, there exists some $\psi: \omega \times \omega \rightarrow \mathbb{Z}$ agreeing mod finite with each function in $\Phi$. More succinctly, in a parlance that has since grown standard, $\lim ^{1} \mathbf{A}=0$ if and every coherent $\Phi$ as above is trivial. By way of this characterisation, Mardešić and Prasolov showed that the continuum hypothesis implies that $\lim ^{1} \mathbf{A} \neq 0$. It follows that it is consistent with the ZFC axioms that strong homology is not additive, not even on the class of closed subspaces of $\mathbb{R}^{2}$.

The following year, Alan Dow, Petr Simon and Jerry Vaughan showed that the Proper Forcing Axiom implies that $\lim ^{1} \mathbf{A}=0$, underscoring the possibility that the additivity of strong homology may also be consistent with the ZFC axioms [9]. Soon thereafter, Stevo Todorcevic showed that the Open Colouring Axiom implies that $\lim ^{1} \mathbf{A}=0$, while Martin's Axiom does not [33, 34]. In 2005, Andrei Prasolov produced a nonmetrisable ZFC counterexample to the additivity of strong homology [27], but the additivity question on any 'nicer' class - Polish or locally compact metric spaces, for example, or even metric spaces outright - remained entirely open (and remains so for the aforementioned classes; see, however, Remark 1.2 for recent progress on this question). ${ }^{1}$ In short, in this sequence the vanishing of $\lim ^{n} \mathbf{A}$ became a topic of set-theoretic study in its own right [12,16], one closely linked to the study of forcing axioms and, furthermore, rekindling older lines of research into relations between homological dimension and the cardinals $\left\{\boldsymbol{\kappa}_{n} \mid n \in \omega\right\}$ [26, 7]. It was therefore natural that the consistency of the additivity of strong homology would close out the list of open questions in Justin Moore's 2010 International Congress of Mathematicians survey on the Proper Forcing Axiom [25]. Moore further observed therein that 'it is entirely possible that it is a theorem of ZFC that either $\left[\lim ^{1} \mathbf{A} \neq 0\right]$ or $\left[\lim ^{2} \mathbf{A} \neq 0\right]$.' The first set-theoretic computation of $\lim ^{2} \mathbf{A}$ appeared some seven years later in [4]; here framings of $\lim ^{n} \mathbf{A}(n>1)$ in terms of higher-dimensional coherence were given and applied to show that the Proper Forcing Axiom implies that $\lim ^{2} \mathbf{A} \neq 0$. Here also Goblot's work [13] was applied to show that $\mathfrak{D} \leq \boldsymbol{\aleph}_{m}$ implies that $\lim ^{n} \mathbf{A}=0$ for all $n>m$. Still, Moore's speculation remained unanswered.

Against this background, we may state our main result:

Main Theorem. Let $\kappa \in V$ be a weakly compact cardinal and let $\mathbb{P}$ denote a length- $\kappa$ finite-support iteration of Hechler forcings. Then

$$
V^{\mathbb{P}} \vDash \lim ^{n} \boldsymbol{A}=0 \text { for all } n>0 . '
$$

In addition to answering well-studied set-theoretic questions, the theorem is of interest for specifying a ZFC model - namely, $V^{\mathbb{P}}$ - in which strong homology may well turn out to be additive on some 'nice' class of spaces properly containing the class of spaces homotopy equivalent to a CW-complex. Moreover, although we have followed tradition in foregrounding the additivity question, the nonvanishing of $\lim ^{n} \mathbf{A}$ for some $n>0$ is also the only known obstacle to strong homology having compact supports on some such 'nice' class; hence this property of strong homology conceivably holds in $V^{\mathbb{P}}$ as well.

${ }^{1}$ Prasolov's example was, in essence, a geometric 'realisation' of an $\left(\omega_{1}, \omega_{1}^{*}\right)$-Hausdorff gap. 
Remark 1.2. In the time since the results in this paper were obtained and the paper was first written, a couple of further developments have occurred that should be noted.

First, work of the first author together with Nathaniel Bannister and Justin Tatch Moore [1] has vindicated the speculations of the previous paragraph by showing that in $V^{\mathbb{P}}$, strong homology is indeed additive and has compact supports on the class of locally compact separable metric spaces. For the property of having compact supports, this class of spaces is at least close to optimal, by the results of [14], but the question of whether strong homology is consistently additive on some broader class of spaces remains open, as we discuss at greater length in our conclusion.

Second, work of the authors together with Michael Hrušák [5], building on the techniques of this paper, shows that the assumption of a weakly compact cardinal is not necessary to obtain the conclusion of our main theorem. More precisely, $\lim ^{n} \mathbf{A}=0$ for all $n>0$ after adding $\beth_{\omega}$-many Cohen reals to any model of ZFC.

For interested readers, the most immediate point is probably the following: [1] and [5] each couple multiple new ideas to techniques which first appear - and consequently receive their fullest and most direct treatment - in the present work. Given the complexity of these techniques, there will be benefits to reading this work before either of the others, though ultimately, of course, readers may and should order their approach to these three texts most fundamentally according to their interests.

These limits also bear on questions seemingly remote from strong homology: within the context of Dustin Clausen and Peter Scholze's recently developed condensed mathematics [28], A is the most basic system in a family of inverse systems $\mathbf{A}_{\kappa, \lambda}$ upon whose limits' simultaneous vanishing the full and faithful embedding of the derived category of pro-abelian groups into the associated derived condensed category depends [8]. More generally speaking, our theorem reflects new levels of insight into the set-theoretic content of derived functors, and thus takes its place in a line of research beginning with Shelah's solution to the Whitehead problem [11,30]. It bears comparison, lastly, with Boban Veličković and Alessandro Vignati's recent result in the opposite direction:

\section{Theorem 1.3 ([35]). For all integers $n \geq 0$, it is consistent with the ZFC axioms that $\lim ^{n} \boldsymbol{A} \neq 0$.}

As in so much of the research history described in the foregoing, our argument of a fundamentally algebraic result will be predominantly set-theoretic in nature. Our Main Theorem, in particular, can and will be recast as a purely set-theoretic statement that we feel is of interest in its own right. For this reason we have divided Section 2, in which we fix our notational conventions and record the definitions and background facts most relevant to our main result, into two subsections. One is more set-theoretic and one is more cohomological in character. Section 2.1 contains the set-theoretic reformulation of our Main Theorem and must be read in order to understand the arguments of subsequent sections. Section 2.2 records some of the original context of the problems addressed in this paper, together with the argument that our set-theoretic reformulation of the Main Theorem is in fact equivalent to its first statement. Readers less familiar with homological algebra may safely skip most of Section 2.2 without sacrificing any understanding of the remainder of the paper (we indicate at the end of Section 2.1 exactly which part of Section 2.2 is necessary for the rest of the paper). Of course, such readers are invited to return to this subsection after reading that remainder, in order to better contextualise its contents.

The paper thereafter is structured as follows. In Section 3 we describe a multidimensional $\Delta$-system lemma lying at the core of our subsequent arguments. We then turn to the proof of our Main Theorem. Due to the technical complexity of the full proof of the theorem, we begin by presenting two cases in which our argument's core ideas appear more transparently. In Section 4, after recording the requisite facts about finite-support iterations of Hechler forcing, we prove the $n=1$ case of our main theorem. We prove the $n=2$ case in Section 5. The full proof of the Main Theorem is contained in Section 6. We conclude with a brief discussion of the import of this result and with the questions following most immediately in its wake.

We close this introduction with a more general word on notations and conventions. For any $X$ and cardinal $\lambda$, we write $[X]^{\lambda}$ to denote the collection of subsets of $X$ of cardinality $\lambda$ and $[X]^{<\lambda}$ for 
$\bigcup_{\kappa<\lambda}[X]^{\kappa}$. In particular, we view $[X]^{0}$ as $\{\emptyset\}$. When $X$ is a collection of ordinals, it is frequently convenient to regard elements $u$ of $[X]^{<\omega}$ as finite increasing sequences, and vice versa. For such $u$ and $i<|u|$, we let $u(i)$ denote the unique $\alpha \in u$ such that $|u \cap \alpha|=i$. The notation $\vec{u}$ will always stand for an ordered tuple of the form $\left\langle u_{0}, \ldots, u_{k}\right\rangle$, though we will occasionally begin our indexing with 1 . Also, if $u, v$ and $w$ are finite sets of ordinals, then the statement $w=u^{\frown} v$ indicates both that $w=u \cup v$ and that $u<v$ - that is, that every ordinal in $u$ is less than every ordinal in $v$ - and the statement $u \sqsubseteq v$ indicates that $u$ is an initial segment of $v$. We use angled brackets to emphasise the indexed or ordered character of a set; in all of this, though, the surest guide will simply be context.

We follow [3] in our approach to forcing. We remark, though, that we view conditions in a $\kappa$-length finite-support iteration as finite partial functions $p$ with domains contained in $\kappa$ rather than as total functions $p$ such that $p(\alpha)$ is a name for the trivial condition for all but finitely many $\alpha<\kappa$. The difference between these views, of course, is cosmetic.

\section{Main definitions and conventions}

\subsection{Set-theoretic background}

Our primary objects of study are families of functions from subsets of $\omega^{2}$ to $\mathbb{Z}$. Let us begin by introducing some basic definitions and notational conventions.

Given functions $f, g: \omega \rightarrow \omega$, set $f \leq g$ if and only if $f(i) \leq g(i)$ for all $i \in \omega ; f \leq^{*} g$ if and only if $f(i) \leq g(i)$ for all but finitely many $i \in \omega$; and $f={ }^{*} g$ if and only if $f(i)=g(i)$ for all but finitely many $i \in \omega$. We let $f \wedge g$ denote the greatest lower $\leq$-bound of $f$ and $g-$ that is, $(f \wedge g)(i)=\min (\{f(i), g(i)\})$ for all $i \in \omega$. Similarly, if $\vec{f}=\left\langle f_{0}, \ldots, f_{n}\right\rangle$ is a sequence of elements of ${ }^{\omega} \omega$, then $\wedge \vec{f}$ denotes the greatest lower $\leq$-bound of the functions $f_{0}, \ldots, f_{n}$. If $f \in{ }^{\omega} \omega$, then $I(f)$ denotes the set $\left\{(i, j) \in \omega^{2} \mid j \leq f(i)\right\}$; visually, this is the region below the graph of $f$. Relatedly, $U(f)$ denotes the set $\left\{g \in{ }^{\omega} \omega \mid g \leq f\right\}$. We note that, for ease of readability, we will sometimes write $\wedge \vec{f}$ in place of the more formally correct $\wedge \vec{f}$ in expressions such as $\varphi: I(\wedge \vec{f}) \rightarrow \mathbb{Z}$. The correct interpretation will always be clear from context.

Our interest is in families of functions indexed by elements of $\left({ }^{\omega} \omega\right)^{n}$ for some positive integer $n$. Before giving general definitions, we recall the better-known special case in which $n=1$.

Definition 2.1. A family of functions $\Phi=\left\langle\varphi_{f}: I(f) \rightarrow \mathbb{Z} \mid f \in{ }^{\omega} \omega\right\rangle$ is coherent if

$$
\varphi_{g} \uparrow I(f \wedge g)-\varphi_{f} \uparrow I(f \wedge g)={ }^{*} 0
$$

for all $f, g \in{ }^{\omega} \omega$.

The family $\Phi$ is trivial if there exists a function $\psi: \omega^{2} \rightarrow \mathbb{Z}$ such that

$$
\psi \uparrow I(f)-\varphi_{f}={ }^{*} 0
$$

for all $f \in{ }^{\omega} \omega$. In this case, we say that $\psi$ trivialises $\Phi$.

The notions of coherent and trivial also apply in the obvious way to families of functions indexed by some subset of ${ }^{\omega} \omega$.

Remark 2.2. In the following, as in Definition 2.1, sums involving functions with different domains will be common. In the interests of readability, we will tend to refrain from notating the restrictions of such functions to the intersection of their domains. An equation like

$$
\varphi_{g} \uparrow I(f \wedge g)-\varphi_{f} \uparrow I(f \wedge g)={ }^{*} 0,
$$

for example, will more typically appear as

$$
\varphi_{g}-\varphi_{f}{ }^{*} 0
$$

hereafter. 
Clearly any trivial family of functions is coherent. More interesting are those families of functions which are coherent but not trivial; in these families there is a tension between local and global behaviours which is exemplary of the broader set-theoretic theme of incompactness. More precisely, observe that any coherent $\Phi=\left\langle\varphi_{f} \mid f \in{ }^{\omega} \omega\right\rangle$ is 'locally' trivial: for any $g \in{ }^{\omega} \omega$, the function $\varphi_{g}$ trivialises the family $\left\langle\varphi_{f} \mid f \in U(g)\right\rangle$. A nontrivial coherent $\Phi$ is simply one in which these local phenomena do not globalise. Such families are the subjects of [9], [34], [16], and [12], among others. As we will see in Section 2.2, their existence is equivalent to the statement $\lim ^{1} \mathbf{A} \neq 0$.

For the more general definitions of $n$-coherence and $n$-triviality, we need some more notation.

Definition 2.3. Suppose that $n$ is a positive integer and $\vec{f}=\left\langle f_{0}, \ldots f_{n}\right\rangle$ is a sequence of length $n+1$. If $i \leq n$, then $\vec{f}^{i}$ denotes the sequence of length $n$ obtained by removing the $i$ th entry of $\vec{f}-$ that is, $\vec{f}^{i}=\left\langle f_{0}, \ldots, f_{i-1}, f_{i+1}, \ldots, f_{n}\right\rangle$.

If $\sigma$ is a permutation of $\langle 0, \ldots, n\rangle$, then $\operatorname{sgn}(\sigma)$ denotes the sign or parity of $\sigma$ (so $\operatorname{sgn}(\sigma)$ is either 1 or -1$)$. We will use the notation $\sigma(\vec{f})$ to denote the sequence $\left\langle f_{\sigma(0)}, \ldots, f_{\sigma(n)}\right\rangle$.

Definition 2.4. Let $n$ be a positive integer, and let

$$
\Phi=\left\langle\varphi_{\vec{f}}: I(\wedge \vec{f}) \rightarrow \mathbb{Z} \mid \vec{f} \in\left({ }^{\omega} \omega\right)^{n}\right\rangle
$$

be a family of functions.

1. $\Phi$ is alternating if

$$
\varphi_{\sigma(\vec{f})}=\operatorname{sgn}(\sigma) \varphi_{\vec{f}}
$$

for every $\vec{f} \in\left({ }^{\omega} \omega\right)^{n}$ and every permutation $\sigma$ of $(0, \ldots, n-1)$.

2. $\Phi$ is $n$-coherent if it is alternating and if

$$
\sum_{i=0}^{n}(-1)^{i} \varphi_{\vec{f}^{i}}={ }^{*} 0
$$

for all $\vec{f} \in\left({ }^{\omega} \omega\right)^{n+1}$. (Note that, in accordance with Remark 2.2, for readability we have omitted the restrictions of the functions in this expression to the intersection of their domains. Formally, each $\varphi_{\vec{f}^{i}}$ should be $\varphi_{\vec{f}^{i}} \uparrow I(\wedge \vec{f})$.)

3. For $n>1, \Phi$ is $n$-trivial if there exists an alternating family

$$
\Psi=\left\langle\psi_{\vec{f}}: I(\wedge \vec{f}) \rightarrow \mathbb{Z} \mid \vec{f} \in\left({ }^{\omega} \omega\right)^{n-1}\right\rangle
$$

such that

$$
\sum_{i=0}^{n-1}(-1)^{i} \psi_{\vec{f}^{i}}={ }^{*} \varphi_{\vec{f}}
$$

for all $\vec{f} \in\left({ }^{\omega} \omega\right)^{n}$. We term such a family an $n$-trivialisation of $\Phi$.

As in the case $n=1$, the notions of alternating, $n$-coherent and $n$-trivial apply in obvious ways to families indexed by $F^{n}$, where $F$ is any subset of ${ }^{\omega} \omega$.

Notice that if $n=1$, then every family $\Phi$ is alternating, and the definition of 1-coherence coincides with that of coherence in Definition 2.1. Similarly, we define 1-triviality to coincide with triviality as defined in Definition 2.1. If the value of $n$ is clear from context, then it may be dropped from the expression $n$-trivial. Also, we will sometimes consider functions $\varphi_{\vec{f}}$ or $\psi_{\vec{f}}$ without explicitly specifying 
their domains or codomains. It is in such cases implicit that these functions have domain $I(\wedge \vec{f})$ and codomain $\mathbb{Z}$.

Observe that if $\Phi$ is alternating, then $\varphi_{\vec{f}}=0$ whenever $\vec{f} \in\left({ }^{\omega} \omega\right)^{n}$ has repeated entries, since if the permutation $\sigma$ induces a simple swapping of two repeated entries in $\vec{f}$, then $\operatorname{sgn}(\sigma)=-1$ and $\sigma(\vec{f})=\vec{f}$ and therefore $\varphi_{\vec{f}}=-\varphi_{\sigma(\vec{f})}=-\varphi_{\vec{f}}$.

Just as when $n=1$, any $n$-trivial family of functions is $n$-coherent. Also just as before, nontrivial $n$-coherent families are exemplary cases of set-theoretic incompactness. This is again because if $\Phi=$ $\left\langle\varphi_{\vec{f}} \mid \vec{f} \in\left({ }^{\omega} \omega\right)^{n}\right\rangle$ is an $n$-coherent family, then for each $g$ in ${ }^{\omega} \omega$ the local family $\left\langle\varphi_{\vec{f}} \mid \vec{f} \in U(g)^{n}\right\rangle$ is $n$-trivial, as witnessed by the collection

$$
\left\langle(-1)^{n+1} \varphi_{\vec{h} \frown\langle g\rangle} \mid \vec{h} \in U(g)^{n-1}\right\rangle .
$$

This fact will contrast with the global structure of any nontrivial such $\Phi$.

We will see in Section 2.2 that for each positive integer $n$, the nonexistence of nontrivial $n$-coherent families of functions is equivalent to the statement $\lim ^{n} \mathbf{A}=0$. Hence our Main Theorem may be rephrased as follows:

Main Theorem (version 2). Let $\kappa$ be a weakly compact cardinal in $V$ and let $\mathbb{P}$ denote a length- $\kappa$ finitesupport iteration of Hechler forcings. Then the following holds in $V^{\mathbb{P}}$ : for every positive integer $n$, every $n$-coherent family

$$
\Phi=\left\langle\varphi_{\vec{f}}: I(\wedge \vec{f}) \rightarrow \mathbb{Z} \mid \vec{f} \in\left({ }^{\omega} \omega\right)^{n}\right\rangle
$$

is n-trivial.

It is this version of the Main Theorem that we will prove. To that end, we give the following alternate characterisation of the $n$-triviality of an $n$-coherent family, one which is at least locally more finitary in character than that of Definition 2.4. Our aim is to facilitate the analysis of these phenomena as they arise in forcing extensions. In what follows, and throughout the paper, if $\psi: X \rightarrow \mathbb{Z}$ is a function, then the support of $\psi$ is the set $\{x \in X \mid \psi(x) \neq 0\}$. We say that $\psi$ is finitely supported if the support of $\psi$ is finite.

Lemma 2.5. Suppose that $F \subseteq{ }^{\omega} \omega$, and let $\Phi=\left\langle\varphi_{\vec{f}} \mid \vec{f} \in F^{n}\right\rangle$ be an n-coherent family. Then $\Phi$ is $n$ trivial if and only if there exists an alternating family of finitely supported functions $\Psi=\left\langle\psi_{\vec{f}} \mid \vec{f} \in F^{n}\right\rangle$ such that

$$
\sum_{i=0}^{n}(-1)^{i} \varphi_{\vec{f}^{i}}=\sum_{i=0}^{n}(-1)^{i} \psi_{\vec{f}^{i}}
$$

for all $\vec{f} \in F^{n+1}$.

Remark 2.6. See Lemma 2.13 for a cohomological approach to this fact; the treatment there is notably cleaner and neatly complements the more computational perspective here.

Proof of Lemma 2.5. We first consider the case in which $n=1$. Suppose that $\Phi$ is trivial and that $\tau: \omega^{2} \rightarrow \mathbb{Z}$ is a trivialisation. For each $f \in F$, let

$$
\psi_{f}=\varphi_{f}-\tau \uparrow I(f)
$$

It is straightforward to verify that $\Psi=\left\langle\psi_{f} \mid f \in F\right\rangle$ is as desired. For the other direction, suppose that $\Psi$ is as in the statement of the lemma. In particular, for all $f, g \in F$ and all $x \in I(f \wedge g)$, we have

$$
\varphi_{f}(x)-\psi_{f}(x)=\varphi_{g}(x)-\psi_{g}(x) .
$$


We can therefore define $\tau: \omega^{2} \rightarrow \mathbb{Z}$ by setting, for all $x \in \omega^{2}$,

$$
\tau(x)=\varphi_{f}(x)-\psi_{f}(x)
$$

for some (or, equivalently, all) $f \in F$ such that $x \in I(f)$. (If there is no $f \in F$ such that $x \in I(f)$, simply let $\tau(x)=0$.) Since each $\psi_{f}(x)$ is finitely supported, it follows that $\varphi_{f}(x)={ }^{*} \tau \uparrow I(f)$ for all $f \in F$.

We now consider the case in which $n>1$. Suppose first that $\Phi$ is $n$-trivial, and let $T=\left\langle\tau_{\vec{f}} \mid \vec{f} \in F^{n-1}\right\rangle$ be an $n$-trivialisation of $\Phi$. For each $\vec{f} \in F^{n}$, let

$$
\psi_{\vec{f}}=\varphi_{\vec{f}}-\sum_{i=0}^{n-1}(-1)^{i} \tau_{\vec{f}^{i}}
$$

We claim that $\Psi=\left\langle\psi_{\vec{f}} \mid \vec{f} \in F^{n}\right\rangle$ is as desired. The following statements are straightforward but tedious to verify; this direction of the lemma being inessential to the proof of our main result, we leave them to the reader:

- If $T$ is an $n$-trivialisation of $\Phi$, then each $\psi_{\vec{f}}$ is finitely supported.

○ If both $T$ and $\Phi$ are alternating families, then $\Psi$ also is an alternating family.

○ For all $\vec{f} \in F^{n}$,

$$
\sum_{i=0}^{n}(-1)^{i} \varphi_{\vec{f}^{i}}=\sum_{i=0}^{n}(-1)^{i} \psi_{\vec{f}^{i}}
$$

as desired. This can be seen by writing

$$
\sum_{i=0}^{n}(-1)^{i} \psi_{\vec{f}^{i}}=\sum_{i=0}^{n}(-1)^{i} \varphi_{\vec{f}^{i}}-\sum_{i=0}^{n} \sum_{j=0}^{n-1}(-1)^{i+j} \tau_{\left(\vec{f}^{i}\right)^{j}}
$$

and verifying that all of the terms in the double sum on the right-hand side cancel out.

For the implication in the other direction, suppose that $\Phi$ and $\Psi$ are as in the statement of the lemma. For each $x \in \omega^{2}$, fix a function $f_{x} \in F$ with $x \in I\left(f_{x}\right)$ if there exists such a function in $F$ (if not, then leave $f_{x}$ undefined). We will define an $n$-trivialisation $T=\left\langle\tau_{\vec{f}} \mid \vec{f} \in F^{n-1}\right\rangle$ of $\Phi$ as follows. Given $\vec{f} \in F^{n-1}$ and $x \in I(\wedge \vec{f})$, note that $f_{x}$ is defined, and let

$$
\tau_{\vec{f}}(x)=(-1)^{n}\left(\psi_{\vec{f}-\left\langle f_{x}\right\rangle}(x)-\varphi_{\vec{f}-\left\langle f_{x}\right\rangle}(x)\right) .
$$

The fact that $T$ is an alternating family follows immediately from the fact that $\Phi$ and $\Psi$ are alternating families. To see that $T$ is an $n$-trivialisation of $\Phi$, fix $\vec{f} \in F^{n}$ and let $x$ be an element of $I(\wedge \vec{f})$ outside of the support of $\psi_{\vec{f}}$. Then we have

$$
\sum_{i=0}^{n-1}(-1)^{i} \tau_{\vec{f}^{i}}(x)=(-1)^{n} \sum_{i=0}^{n-1}(-1)^{i}\left(\psi_{\vec{f}^{i}-\left\langle f_{x}\right\rangle}(x)-\varphi_{\vec{f}^{i}-\left\langle f_{x}\right\rangle}(x)\right) .
$$

Letting $\vec{g}=\vec{f}^{\frown}\left\langle f_{x}\right\rangle$, we have

$$
\sum_{i=0}^{n}(-1)^{i} \varphi_{\vec{g}^{i}}(x)=\sum_{i=0}^{n}(-1)^{i} \psi_{\vec{g}^{i}}(x) .
$$


Rearranging the terms in this equation yields

$$
\sum_{i=0}^{n-1}(-1)^{i}\left(\psi_{\vec{f}^{i} \backslash\left\langle f_{x}\right\rangle}(x)-\varphi_{\vec{f}^{i} \backslash\left\langle f_{x}\right\rangle}(x)\right)=(-1)^{n}\left(\varphi_{\vec{f}}(x)-\psi_{\vec{f}}(x)\right) .
$$

Recall that we chose $x$ so that $\psi_{\vec{f}}(x)=0$. Putting this all together, we obtain

$$
\sum_{i=0}^{n-1}(-1)^{i} \tau_{\vec{f}^{i}}(x)=(-1)^{2 n} \varphi_{\vec{f}}(x)=\varphi_{\vec{f}}(x) .
$$

Since the support of $\psi_{\vec{f}}$ is finite, it follows that

$$
\sum_{i=0}^{n-1}(-1)^{i} \tau_{\vec{f}^{i}}={ }^{*} \varphi_{\vec{f}}
$$

as required.

With the aid of one further lemma, we are now in a position to describe the basic strategy of the proof of our Main Theorem. When $F$ is a subset of ${ }^{\omega} \omega$ and $\Phi=\left\langle\varphi_{\vec{f}} \mid \vec{f} \in\left({ }^{\omega} \omega\right)^{n}\right\rangle$, we will write $\Phi \uparrow F$ for $\left\langle\varphi_{\vec{f}} \mid \vec{f} \in F^{n}\right\rangle$. Recall that a subset $F \subseteq{ }^{\omega} \omega$ is said to be $\leq{ }^{*}$-cofinal if, for all $g \in{ }^{\omega} \omega$, there is $f \in F$ such that $g \leq^{*} f$.

Lemma 2.7. Let $\Phi=\left\langle\varphi_{\vec{f}} \mid \vec{f} \in\left({ }^{\omega} \omega\right)^{n}\right\rangle$ be an $n$-coherent family of functions. Then $\Phi$ is $n$-trivial if and only if $\Phi \uparrow F$ is n-trivial for some $\leq{ }^{*}$-cofinal $F \subseteq{ }^{\omega} \omega$.

Proof. The rightward implication is obvious. For the leftward implication, the point is that any $n$ trivialisation of any such $\Phi \uparrow F$ extends to an $n$-trivialisation of $\Phi$. Suppose first that $n=1, F$ is $\leq^{*}$-cofinal in ${ }^{\omega} \omega$ and $\psi: \omega^{2} \rightarrow \mathbb{Z}$ trivialises $\Phi \uparrow F$. We claim that $\psi$ trivialises the entire family $\Phi$. Indeed, if $g \in{ }^{\omega} \omega$, then we can find $f \in F$ such that $g \leq^{*} f$ - that is, $I(f)$ contains $I(g)$ modulo a finite set. By the coherence of $\Phi$, we have $\varphi_{f}=^{*} \varphi_{g}$. Since $\psi$ trivialises $\Phi \uparrow F$, we have $\psi={ }^{*} \varphi_{f}$, and hence $\psi={ }^{*} \varphi_{g}$, as well.

We can therefore suppose that $n>1$. Let $F$ be $\leq^{*}$-cofinal in ${ }^{\omega} \omega$ and let $\Upsilon=\left\langle v_{\vec{f}} \mid \vec{f} \in F^{n-1}\right\rangle$ be an $n$-trivialisation of $\Phi \uparrow F$. Fix a map $a:{ }^{\omega} \omega \rightarrow F$ such that $g \leq{ }^{*} a(g)$ for each $g \in{ }^{\omega} \omega$. Write $a(\vec{g})$ for $\left\langle a\left(g_{0}\right), \ldots, a\left(g_{n-1}\right)\right\rangle$. The family $\Psi=\left\langle\psi_{\vec{g}} \mid \vec{g} \in\left({ }^{\omega} \omega\right)^{n-1}\right\rangle$ is then an $n$-trivialisation of $\Phi$, where

$$
\psi_{\vec{g}}=v_{a(\vec{g})}-\sum_{i=0}^{n-1}(-1)^{i} \varphi_{\left\langle g_{0}, \ldots, g_{i}, a\left(g_{i}\right), \ldots, a\left(g_{n-1}\right)\right\rangle}
$$

for each $\vec{g} \in\left({ }^{\omega} \omega\right)^{n-1}$. The right-hand side of the equation is a sum of functions defined on all but finitely many elements of $I(\wedge \vec{g})$; the function $\psi_{\vec{g}}$ is defined more precisely as the extension of that sum to the domain $I(\wedge \vec{g})$ by letting $\psi_{\vec{g}}(x)=0$ on any otherwise undefined arguments $x$. The verification that this definition works takes the following shape: for any $\vec{g}=\left\langle g_{0}, \ldots, g_{n}\right\rangle \in\left({ }^{\omega} \omega\right)^{n+1}$, the terms $(-1)^{i} v_{a\left(\vec{g}^{i}\right)}(i \leq n)$ in

$$
\sum_{i=0}^{n}(-1)^{i} \psi_{\vec{g}^{i}}
$$

together simplify (mod finite) to $\varphi_{a(\vec{g})}$. This together with $(n-1)$ of the terms in sum (1) will simplify (mod finite) to $\varphi\left\langle g_{0}, a\left(g_{1}\right), \ldots, a\left(g_{n-1}\right)\right\rangle$. This process continues until expression (1) has entirely simplified (mod finite) to the term $\varphi_{\vec{g}}$, thus completing the verification. 
There are in fact a number of ways to argue Lemma 2.7. The foregoing approach may be clearer in the following example.

Example 2.8. Let $\Phi$ be 2-coherent. Let $F$ be as in the statement of Lemma 2.7 and let $\Upsilon=\left\langle v_{f} \mid f \in F\right\rangle$ 2-trivialise $\Phi \uparrow F$. Let then $a:{ }^{\omega} \omega \rightarrow F$ be as in the proof of Lemma 2.7 and let $\psi_{f}=v_{a(f)}-\varphi_{\langle f, a(f)\rangle}$ for each $f \in{ }^{\omega} \omega$. Then for all $f, g \in{ }^{\omega} \omega$,

$$
\begin{aligned}
\psi_{g}-\psi_{f} & =v_{a(g)}-v_{a(f)}-\left(\varphi_{\langle g, a(g)\rangle}-\varphi_{\langle f, a(f)\rangle}\right) \\
& ={ }^{*} \varphi_{\langle a(f), a(g)\rangle}-\varphi_{\langle g, a(g)\rangle}+\varphi_{\langle f, a(f)\rangle} \\
& ={ }^{*} \varphi_{\langle f, a(g)\rangle}-\varphi_{\langle g, a(g)\rangle} \\
& ={ }^{*} \varphi_{\langle f, g\rangle}
\end{aligned}
$$

as desired.

We may now describe the broad outlines of our proof of our Main Theorem. Fix $n>0$ and a weakly compact cardinal $\kappa$, and let $\mathbb{P}$ be a length- $\kappa$ finite-support iteration of Hechler forcings. To show that $V^{\mathbb{P}} \vDash$ ' $\lim ^{n} \mathbf{A}=0$ ', we fix an arbitrary $n$-coherent $\Phi$ in $V^{\mathbb{P}}$ and show that it is $n$-trivial. This we accomplish by showing that $\Phi \uparrow F$ is $n$-trivial, where $F$ is a $\leq^{*}$-cofinal family of Hechler reals. This in turn we achieve by defining a family $\left\langle\psi_{\vec{f}} \mid \vec{f} \in F^{n}\right\rangle$ of finitely supported functions trivialising $\Phi \uparrow F$ in the sense of Lemma 2.5. These $\psi_{\vec{f}}$ are formed out of the differences among particular functions $\varphi_{\vec{f}}$ in $\Phi$. To select those functions we rely heavily on uniformities among the conditions in the $\mathbb{P}$-generic filter which derive from the weak compactness of $\kappa$ by way of Lemma 3.4, a multidimensional version of the classical $\Delta$-system lemma. Our proof consists essentially in applying this procedure to each $n>0$. As indicated, however, expository considerations lead us to break this proof into three stages: $n=1, n=2$ and $n>2$.

\subsection{Homological background}

We described above a historical sequence of investigations into the derived limits $\lim ^{n} \mathbf{A}$, the point of departure for any of which is the following computation. For each positive integer $n$, let $X^{n}$ denote the one-point compactification of an infinite countable sum of copies of the $n$-dimensional open unit ball $B^{n}=\left\{\vec{x} \in \mathbb{R}^{n} \mid\|\vec{x}\|<1\right\}$; more colloquially, $X^{n}$ is an $n$-dimensional 'Hawaiian earring.' Its strong homology groups are as follows:

$$
\bar{H}_{p}\left(X^{n}\right)= \begin{cases}\mathbb{Z}, & p=0 \\ \prod_{i \in \omega} \mathbb{Z}, & p=n \\ 0, & \text { otherwise }\end{cases}
$$

This is in marked contrast to the singular homology groups of $X^{n}$ [10], [2], and essentially certifies $\bar{H}_{*}$ as a Steenrod homology theory [24]. For countably infinite sums of copies of $X^{n}$, on the other hand,

$$
\bar{H}_{p}\left(\coprod_{i \in \omega} X^{n}\right)= \begin{cases}\lim ^{n} \mathbf{A} \oplus\left(\bigoplus_{i \in \omega} \mathbb{Z}\right), & p=0, \\ \lim ^{n-p} \mathbf{A}, & 0<p \leq n, \\ 0, & \text { otherwise. }\end{cases}
$$

We will define the inverse system $\mathbf{A}$ and its higher derived limits in a moment; we will then leave all more direct references to strong homology behind until our conclusion. Interested readers are referred to [22] or to [21] more generally for details of these computations. The significance of these computations, of course, is that they tell us that if strong homology is additive on closed subsets of Euclidean space, then $\lim ^{n} \mathbf{A}=0$ for all $n>0$. 
Let $\mathcal{N}$ denote the partial order $\left({ }^{\omega} \omega, \leq\right)$, and let $\mathcal{N}$ op denote its order reversal. Let $\tau(\mathcal{N})$ denote the topology on ${ }^{\omega} \omega$ generated by the basis $\mathrm{B}(\mathcal{N})=\left\{U(g) \mid g \in{ }^{\omega} \omega\right\}$, where we recall that $U(g)=\{f \in$ $\left.{ }^{\omega} \omega \mid f \leq g\right\}$. Given a quotient $B / A$ of groups, we write $[b]$ for the coset of an element $b$ of $B$.

Definition 2.9. Let $\mathbf{A}$ denote the inverse system $\left(A_{f}, p_{f g}, \mathcal{N}\right)$, where

$$
A_{f}=\bigoplus_{i \in I(f)} \mathbb{Z}
$$

and $p_{f g}: A_{g} \rightarrow A_{f}$ is the projection map for each $f \leq g$ in $\mathcal{N}$. Similarly, let $\mathbf{B}=\left(B_{f}, q_{f g}, \mathcal{N}\right)$ and $\mathbf{B} / \mathbf{A}=\left(B_{f} / A_{f}, r_{f g}, \mathcal{N}\right)$, where

$$
B_{f}=\prod_{i \in I(f)} \mathbb{Z}
$$

and $q_{f g}: B_{g} \rightarrow B_{f}$ and $r_{f g}: B_{g} / A_{g} \rightarrow B_{f} / A_{f}$ are projection maps for each $f \leq g$ in $\mathcal{N}$.

$\mathbf{A}$ is an object, in other words, of the functor or presheaf category $A b^{\mathcal{N}^{\text {po }}}$, and it is in this setting that its higher derived limits are most naturally defined. The inverse limit of $\mathbf{A}$, for example, admits both a category-theoretic characterisation via a universal property in $\mathrm{Ab}^{\mathcal{N}}{ }^{\mathrm{op}}$ and the following more concrete characterisation:

$$
\lim \mathbf{A}=\left\{\left\langle\varphi_{f} \mid f \in{ }^{\omega} \omega\right\rangle \in \prod_{f \in \omega^{\omega}} A_{f} \mid p_{f g}\left(\varphi_{g}\right)=\varphi_{f} \text { for all } f \leq g \text { in }{ }^{\omega} \omega\right\} .
$$

More precisely, lim A is the foregoing family naturally viewed as a group. ${ }^{2}$ Similarly, higher derived limits $\lim ^{n} \mathbf{A}$ admit abelian category-theoretic description as the cohomology groups of the lim-image of an injective resolution of $\mathbf{A}$. Standard resolutions convert this description to a more concrete general form, as before. The characterisations of $\lim ^{1} \mathbf{A}$ and of $\lim ^{n} \mathbf{A}(n \geq 1)$ in [22] and [4], respectively, then each involve one further conversion, via the long exact sequence

$$
0 \longrightarrow \lim \mathbf{A} \longrightarrow \lim \mathbf{B} \longrightarrow \lim \mathbf{B} / \mathbf{A} \stackrel{\partial^{0}}{\longrightarrow} \lim ^{1} \mathbf{A} \longrightarrow \lim ^{1} \mathbf{B} \longrightarrow \cdots
$$

associated to the natural short exact sequence

$$
0 \longrightarrow \mathbf{A} \longrightarrow \mathbf{B} \longrightarrow \mathbf{B} / \mathbf{A} \longrightarrow 0 .
$$

For our later results, we will require two further reformulations.

First, for each $U$ in $\tau(\mathcal{N})$ let

$$
\lim (\mathbf{A} \uparrow U)=\left\{\left\langle\varphi_{f} \mid f \in U\right\rangle \in \prod_{f \in U} A_{f} \mid p_{f g}\left(\varphi_{g}\right)=\varphi_{f} \text { for all } f \leq g \text { in } U\right\} .
$$

Observe that

$$
\lim (\mathbf{A} \uparrow U(g)) \cong A_{g} \text { for any } g \in{ }^{\omega} \omega
$$

Similar definitions and observations apply for the systems $\mathbf{B}$ and $\mathbf{B} / \mathbf{A}$. By an observation of Jensen [15, p. 4],

$$
\lim ^{n} \mathbf{A} \cong \check{H}^{n}\left(\left({ }^{\omega} \omega, \tau(\mathcal{N})\right), \mathcal{A}\right)
$$

\footnotetext{
${ }^{2}$ Observe that, in consequence, $\lim ^{0} \mathbf{A}$ - which equals $\lim \mathbf{A}$ by definition - is isomorphic to $\bigoplus_{i \in \omega} \prod_{j \in \omega} \mathbb{Z}$, as additivity in the degree $n=p$ in equation (2) would require.
} 
where $\mathcal{A}$ is the sheaf on $\left({ }^{\omega} \omega, \tau(\mathcal{N})\right)$ given by $U \mapsto \lim (\mathbf{A} \uparrow U)$ together with the natural restriction maps. Analogous definitions apply for the systems $\mathbf{B}$ and $\mathbf{B} / \mathbf{A}$; in particular, $\mathcal{B} / \mathcal{A}$ will denote the sheaf given by $U \mapsto \lim (\mathbf{B} / \mathbf{A} \uparrow U)$. Since $\mathrm{B}(\mathcal{N})$ is maximal in the refinement ordering of open covers of $\mathcal{N}$, expression (3) now takes the following form:

$$
\cdots \rightarrow \check{H}^{0}(\mathrm{~B}(\mathcal{N}), \mathcal{B}) \rightarrow \check{H}^{0}(\mathrm{~B}(\mathcal{N}), \mathcal{B} / \mathcal{A}) \stackrel{\partial^{0}}{\rightarrow} \check{H}^{1}(\mathrm{~B}(\mathcal{N}), \mathcal{A}) \rightarrow \check{H}^{1}(\mathrm{~B}(\mathcal{N}), \mathcal{B}) \rightarrow \cdots
$$

As observed in [4],

$$
\lim ^{n} \mathbf{B}=0 \text { for all } n>0 .
$$

The same therefore holds for $\check{H}^{n}(\mathrm{~B}(\mathcal{N}), \mathcal{B})$. In consequence,

$$
\lim ^{1} \mathbf{A} \cong \frac{\check{H}^{0}(\mathrm{~B}(\mathcal{N}), \mathcal{B} / \mathcal{A})}{\operatorname{im}\left(\check{H}^{0}(\mathrm{~B}(\mathcal{N}), \mathcal{B})\right)} .
$$

We will define these cohomology groups more explicitly below. It will then be clear that the natural rendering of this quotient is as the coherent families of functions modulo the trivial families of functions in the sense of Definition 2.1. The following is then immediate:

Theorem 2.10 ([22]). $\lim ^{1} \boldsymbol{A}=0$ if and only if every coherent family of functions $\left\langle\varphi_{f} \mid f \in{ }^{\omega} \omega\right\rangle$ is trivial.

By equation (5), the higher- $n$ analogue of equation (6) is simply

$$
\lim ^{n} \mathbf{A} \cong \check{H}^{n-1}(\mathrm{~B}(\mathcal{N}), \mathcal{B} / \mathcal{A}) \text { for all } n>1 .
$$

The advantage of this formulation is that in computing Čech cohomology groups we have a choice among several indexing schemas that give equivalent quotients [29, I.20 Proposition 2]. As a generic real may fail to integrate nontrivially into the ordering $\left({ }^{\omega} \omega, \leq\right)$ of its ground model, it is useful in contexts like ours to define higher coherence via indices as untethered to that order as possible. To that end we adopt the alternating cochains definition of the Čech complex.

Definition 2.11. For $n \geq 0$, the group $\check{H}^{n}(\mathrm{~B}(\mathcal{N}), \mathcal{B} / \mathcal{A})$ is the cohomology of the cochain complex

$$
\cdots \stackrel{d^{n-1}}{\longrightarrow} C_{\text {alt }}^{n}(\mathrm{~B}(\mathcal{N}), \mathcal{B} / \mathcal{A}) \stackrel{d^{n}}{\longrightarrow} C_{\text {alt }}^{n+1}(\mathrm{~B}(\mathcal{N}), \mathcal{B} / \mathcal{A}) \stackrel{d^{n+1}}{\longrightarrow} \cdots,
$$

where $C_{\text {alt }}^{n}(\mathrm{~B}(\mathcal{N}), \mathcal{B} / \mathcal{A})$ denotes the subgroup of

$$
\prod_{\vec{f} \in(\omega \omega)^{n+1}} \lim (\mathbf{B} / \mathbf{A} \uparrow U(\wedge \vec{f}))=\prod_{\vec{f} \in(\omega \omega)^{n+1}} B_{\wedge \vec{f}} / A_{\wedge \vec{f}}
$$

whose elements $c$ satisfy

$$
c(\sigma(\vec{f}))=\operatorname{sgn}(\sigma) c(\vec{f})
$$

for all $\vec{f} \in\left({ }^{\omega} \omega\right)^{n+1}$ and permutations $\sigma$ of $\langle 0, \ldots, n\rangle$. The equality in equation (9) amounts simply to an application of the $\mathbf{B} / \mathbf{A}$-variant of equation (4).

The differentials $d^{n}$ are defined as usual. Namely, for any $i \leq n+1$ and $\vec{f} \in\left({ }^{\omega} \omega\right)^{n+2}$, recall that $\vec{f}^{i}$ denotes the element of $\left({ }^{\omega} \omega\right)^{n+1}$ formed via the omission of the coordinate $f_{i}$ from $\vec{f}$. Then for any $c \in C_{\text {alt }}^{n}(\mathrm{~B}(\mathcal{N}), \mathcal{B} / \mathcal{A})$, define $d^{n} c$ by the assignment

$$
d^{n} c(\vec{f})=\sum_{i=0}^{n+1}(-1)^{i}\left(c\left(\vec{f}^{i}\right) \uparrow I(\wedge \vec{f})\right)
$$

for each $\vec{f} \in\left({ }^{\omega} \omega\right)^{n+2}$. 
Entirely analogous definitions hold for $\check{H}^{n}(\mathrm{~B}(\mathcal{N}), \mathcal{A})$ and $\check{H}^{n}(\mathrm{~B}(\mathcal{N}), \mathcal{B})$. Observe in particular that any $c$ in $C_{\text {alt }}^{n}(\mathrm{~B}(\mathcal{N}), \mathcal{B} / \mathcal{A})$ admits representatives $\hat{c}$ in $C_{\text {alt }}^{n}(\mathrm{~B}(\mathcal{N}), \mathcal{B})$ - that is, alternating cochains $\hat{c}$ taking values in the groups $B \wedge \vec{f}$ and satisfying $[\hat{c}(\vec{f})]=c(\vec{f})$ for all $\vec{f} \in\left({ }^{\omega} \omega\right)^{n+1}$.

As in the cases of Section 2.1, the requisite function restrictions will tend to be clear from context. Hence again for readability, we will rarely notate them, writing, for example, sums like that of equation (10) as

$$
\sum_{i=0}^{n+1}(-1)^{i} c\left(\vec{f}^{i}\right) .
$$

It is now clear that $n$-coherent and $n$-trivial families are representatives of, respectively, the $(n-1)$ cocycles and $(n-1)$-coboundaries of complex (8). Recall that every $n$-trivial $\Phi$ is $n$-coherent. We have just argued that whether or not $\lim ^{n} \mathbf{A}$ vanishes is precisely the question of the converse; we have in other words shown the following:

Theorem 2.12. Let $n$ be a positive integer. Then $\lim ^{n} \boldsymbol{A}=0$ if and only if every $n$-coherent family $\Phi=\left\langle\varphi_{\vec{f}} \mid \vec{f} \in\left({ }^{\omega} \omega\right)^{n}\right\rangle$ is n-trivial.

We end this subsection by indicating how Lemma 2.5 can be derived from cohomological considerations in the special case $F={ }^{\omega} \omega$. (The more general case of an arbitrary $F \subseteq{ }^{\omega} \omega$ can be obtained via a routine modification of the arguments in this subsection, working with the partial order $\mathcal{N}_{F}=(F, \leq)$ and inverse systems $\mathbf{A} \uparrow F=\left(A_{f}, p_{f g}, \mathcal{N}_{F}\right), \mathbf{B} \uparrow F$ and $\mathbf{B} / \mathbf{A} \uparrow F$ instead of $\mathcal{N}, \mathbf{A}, \mathbf{B}$ and $\mathbf{B} / \mathbf{A}$. All of the results of this subsection carry through with no difficulty in this setting.) We return to equation (7), which was derived from the cohomological reformulation of expression (3). Equation (7) is more precisely stated as follows:

Lemma 2.13. Let $n$ be greater than 1 . Then $\check{H}^{n-1}(\mathrm{~B}(\mathcal{N}), \mathcal{B} / \mathcal{A})$ is isomorphic to $\check{H}^{n}(\mathrm{~B}(\mathcal{N})$, $\mathcal{A})$ via the connecting map $\partial_{n-1}:[c] \mapsto\left[d^{n-1} \hat{c}\right]$, where $\hat{c}$ is any representative of $c$ in $C_{\text {alt }}^{n-1}(\mathrm{~B}(\mathcal{N}), \mathcal{B})$.

Proof. Here the only new assertion is the characterisation of the connecting map $\partial_{n-1}$. This, though, is simply the general form of the connecting maps of a long exact sequence; see, for example, [36, Addendum 1.3.3].

For $n \geq 1$ it follows that an $n$-coherent family $\Phi=\left\langle\varphi_{\vec{f}} \mid \vec{f} \in\left({ }^{\omega} \omega\right)^{n}\right\rangle$ represents a coboundary in $C_{\text {alt }}^{n}(\mathrm{~B}(\mathcal{N}), \mathcal{B} / \mathcal{A})$ if and only if what we might write as $d^{n} \Phi$ is a coboundary in $C_{\text {alt }}^{n+1}(\mathrm{~B}(\mathcal{N}), \mathcal{A})$. Lemma 2.5 is then immediate.

\section{A multidimensional $\Delta$-system lemma}

We turn now more toward the argument of our Main Theorem. We begin by proving a generalisation of the 2-dimensional $\Delta$-system lemma appearing in [6] (see [32] for an earlier precedent). Structuring our generalisation will be the following refinement of the relation holding between pairs of elements of a classical $\Delta$-system:

Definition 3.1. Sets $u, v \in[\text { Ord }]^{<\omega}$ are aligned if

$\circ|u|=|v|$ and

○ $|u \cap \alpha|=|v \cap \alpha|$ for all $\alpha \in u \cap v$.

Definition 3.2. Let $0<k<\ell$ be natural numbers. A function $t: \ell \rightarrow 3$ is a type of width $k$ and length $\ell$ if

$$
\left|t^{-1}[\{0,2\}]\right|=\left|t^{-1}[\{1,2\}]\right|=k .
$$


Given $u, v \in[\mathrm{Ord}]^{k}$ and an increasing enumeration $\left\langle\alpha_{i}\right| i\langle\ell\rangle$ of $u \cup v$, we say that the type of $u$ and $v$ is the unique type $t$ of width $k$ and length $\ell$ such that

$\circ u \backslash v=\left\{\alpha_{i} \mid t(i)=0\right\}$,

$\circ v \backslash u=\left\{\alpha_{i} \mid t(i)=1\right\}$ and

○ $u \cap v=\left\{\alpha_{i} \mid t(i)=2\right\}$.

We denote this unique type $t$ by $\operatorname{tp}(u, v)$. Observe that $u$ and $v$ are aligned if and only if $t=\operatorname{tp}(u, v)$ satisfies

$$
\left|t^{-1}[\{0\}] \cap i\right|=\left|t^{-1}[\{1\}] \cap i\right|
$$

for all $i$ in $t^{-1}[\{2\}]$. Any such type $t$ is also called aligned.

These types admit natural representations as finite strings of 0 s, 1 s and 2 s. If $\alpha<\beta<\gamma$, for example, then $\operatorname{tp}(\{\alpha, \beta\},\{\beta, \gamma\})$ is naturally rendered as 021 .

We may now state our multidimensional $\Delta$-system lemma. We first remind the reader of the definition of a weakly compact cardinal.

Definition 3.3. A cardinal $\kappa$ is weakly compact if it is uncountable and, for every positive integer $n$, every $\theta<\kappa$ and every function $c:[\kappa]^{n} \rightarrow \theta$, there is a set $H \in[\kappa]^{\kappa}$ such that $c \uparrow[H]^{n}$ is constant.

There are many useful alternative characterisations of weakly compact cardinals; for example, $\kappa$ is weakly compact if and only if it is strongly inaccessible and has the tree property. We refer the reader to [17] for more information on this and other equivalent formulations of weak compactness.

Recall our notational convention that elements of $[\kappa]^{n}$ are often thought of as increasing $n$-tuples. So, for instance, when we write $\vec{\alpha}=\left\langle\alpha_{0}, \ldots, \alpha_{n-1}\right\rangle \in[\kappa]^{n}$, it is implicit that $\alpha_{0}<\alpha_{1}<\cdots<\alpha_{n-1}$.

Lemma 3.4. Let $n$ be a positive number and let $\kappa$ be a weakly compact cardinal, and suppose that the family of $n$-tuples $\vec{\alpha}=\left\langle\alpha_{0}, \ldots, \alpha_{n-1}\right\rangle \in[\kappa]^{n}$ indexes elements $u_{\vec{\alpha}}$ of $[\kappa]^{<\omega}$. Then there is a set $A \in[\kappa]^{\kappa}$ and a collection $\left\langle u_{\vec{\alpha}} \mid \vec{\alpha} \in[A]^{<n}\right\rangle$ of elements of $[\kappa]^{<\omega}$ such that the following are true:

1. For all $\vec{\alpha} \in[A]^{<n}$,

(a) if $\beta \in A$ and $\vec{\alpha}<\beta$, then $u_{\vec{\alpha}}<\beta$;

(b) if $\vec{\beta} \in[A] \leq n$ satisfies $\vec{\alpha} \sqsubseteq \vec{\beta}$, then $u_{\vec{\alpha}} \sqsubseteq u_{\vec{\beta}}$;

(c) the set $\left\{u_{\vec{\alpha}}-\langle\beta\rangle \mid \beta \in A \backslash(\max (\vec{\alpha})+1)\right\}$ forms a $\Delta$-system with root $u_{\vec{\alpha}}$.

2. For all $m \leq n$ and all $\vec{\alpha}, \vec{\beta} \in[A]^{m}$,

(a) $\left|u_{\vec{\alpha}}\right|=\left|u_{\vec{\beta}}\right|$ and

(b) if $\vec{\alpha}$ and $\vec{\beta}$ are aligned, then $u_{\vec{\alpha}}$ and $u_{\vec{\beta}}$ are aligned.

To prove Lemma 3.4, we require one further technical lemma.

Lemma 3.5. Suppose that $0<k<\ell<\omega$ and that $t$ is an aligned type of width $k$ and length $\ell$. Then for some positive number $m$ there exists a sequence $\left\langle u_{j} \mid j \leq 2 m\right\rangle$ of elements of [Ord] ${ }^{k}$ such that

$\circ \operatorname{tp}\left(u_{2 j}, u_{2 j+1}\right)=\operatorname{tp}\left(u_{2 j+2}, u_{2 j+1}\right)=t$ for all $j<m$ and

$\circ \operatorname{tp}\left(u_{0}, u_{2 m}\right)=t$.

Proof. The proof is by induction on $k$. We will also assume that $t(\ell-1) \in\{1,2\}$. The case in which $t(\ell-1)=0$ is essentially the same as that in which $t(\ell-1)=1$, and is left to the reader. If $k=1$, then either $t=2$ or $t=01$. We may take $m=1$ in either case, for if $t=2$, then for any ordinal $\alpha$ the sequence $u_{0}=u_{1}=u_{2}=\{\alpha\}$ is as desired, whereas if $t=01$, then for any ordinals $\alpha<\beta<\gamma$ the sequence $u_{0}=\{\alpha\}, u_{1}=\{\gamma\}, u_{2}=\{\beta\}$ is as desired.

Now assume that $k>1$ and that we have established the lemma for all aligned types of width less than $k$.

Case 1: $t(\ell-1)=2$. In this case, $t^{\prime}=t \uparrow(\ell-1)$ is an aligned type of width $k-1$, hence by our inductive hypothesis there exists some positive $m$ and sequence $\left\langle u_{j} \mid j \leq 2 m\right\rangle$ of elements of [Ord] ${ }^{k-1}$ 
witnessing the conclusion of the lemma for $t^{\prime}$. Now take any $\beta>\cup_{j \leq 2 m} u_{j}$, and let $u_{j}^{\star}=u_{j} \cup\{\beta\}$ for all $j$. Then $m$ and $\left\langle u_{j}^{\star} \mid j \leq 2 m\right\rangle$ witness the conclusion of the lemma for $t$.

Case 2: $t(\ell-1)=1$. The idea in this case is to form a type $t^{\prime}$ of width $k-1$ and length $\ell-2$ by deleting the last 0 and the last 1 from $t$. The induction hypothesis then furnishes a sequence $\left\langle u_{j} \mid j \leq 2 m\right\rangle$ as in the conclusion of the lemma for the type $t^{\prime}$. It is then fairly straightforward (though notationally tedious) to modify $\left\langle u_{j} \mid j \leq 2 m\right\rangle$ to form a sequence as desired for the original type $t$.

More formally, let $\ell_{0}<\ell$ be such that $t\left(\ell_{0}\right)=0$ and $t(i)=1$ for all $i$ with $\ell_{0}<i<\ell$. Such an $\ell_{0}$ must exist, since $t$ is aligned. Let $k_{0}=\left|t^{-1}[\{1,2\}] \cap \ell_{0}\right|$. This number $k_{0}$ has the following significance: if $u, v \in[\text { Ord }]^{k}$ are such that $\operatorname{tp}(u, v)=t$, then $k_{0}$ is the number of elements of $v$ that are smaller than the largest element of $u$. In other words, $v\left(k_{0}\right)$ is the least element of $v$ larger than every element of $u$. Now define $t^{\prime}:(\ell-2) \rightarrow 3$ by letting

$$
t^{\prime}(i)= \begin{cases}t(i) & \text { if } i<\ell_{0} \\ 1 & \text { if } \ell_{0} \leq i<\ell-2\end{cases}
$$

This defines an aligned type $t^{\prime}$ of width $k-1$. By applying the induction hypothesis, we obtain a positive $m$ and a sequence $\left\langle u_{j} \mid j \leq 2 m\right\rangle$ of elements of [Ord] ${ }^{k-1}$ witnessing the conclusion of the lemma for $t^{\prime}$. By 'stretching' each $u_{j}$ if necessary, we may assume that every element of every $u_{j}$ is a limit of limit ordinals.

We now augment the elements of the sequence $\left\langle u_{j} \mid j \leq 2 m\right\rangle$ to form a sequence $\left\langle u_{j}^{\star} \mid j \leq 2 n\right\rangle$ for $t$. Two cases are straightforward: $k_{0}=0$ and $k_{0}=k-1$. In the latter case, for example, take $\left\{\beta_{j} \mid j \leq 2 m\right\}>\bigcup_{j \leq 2 m} u_{j}$ such that

$\circ$ for all $j, j^{\prime} \leq 2 m$, if $j$ is even and $j^{\prime}$ is odd, then $\beta_{j}<\beta_{j^{\prime}}$; and ○ $\beta_{0}<\beta_{2 m}$.

Letting $u_{j}^{\star}=u_{j} \cup\left\{\beta_{j}\right\}$ then defines a sequence as desired for $t$. The case of $k_{0}=0$ is similar. Therefore, assume that $0<k_{0}<k-1$. We will assume as well that $u_{j}\left(k_{0}\right)>u_{j^{\prime}}\left(k_{0}-1\right)$ for all odd $j, j^{\prime}<2 m$. We are free to assume this because if $\operatorname{tp}(u, v)=t^{\prime}$, then $v\left(k_{0}\right)$ is larger than every element of $u$, hence $\operatorname{tp}(u, w)=t^{\prime}$ for any $w$ with $w \uparrow k_{0}=v \uparrow k_{0}$ and $w \uparrow\left[k_{0}, k-1\right)$ consisting of arbitrarily large ordinals.

We now define limit ordinals $\left\langle\beta_{j} \mid j \leq 2 m\right\rangle$ so that $u_{j}<\beta_{j}$ for each $j \leq 2 m$. We first do this for the even $j \leq 2 m$. First let $\beta_{0}$ be a limit ordinal such that $u_{1}\left(k_{0}-1\right)<\beta_{0}<u_{1}\left(k_{0}\right)$, and let $\beta_{2 m}$ be a limit ordinal such that $u_{2 m-1}\left(k_{0}-1\right)<\beta_{2 m}<u_{2 m-1}\left(k_{0}\right)$. For any other even $0<j<2 m$, let $\beta_{j}>u_{j}$ be a limit ordinal satisfying

$$
\max \left\{u_{j-1}\left(k_{0}-1\right), u_{j+1}\left(k_{0}-1\right)\right\}<\beta_{j}<\min \left\{u_{j-1}\left(k_{0}\right), u_{j+1}\left(k_{0}\right)\right\} .
$$

This is all possible by the assumptions of the preceding paragraphs.

Lastly, let $\beta^{\star}$ be a limit ordinal larger than any ordinal so far considered, and for all odd $j<2 m$ let $\beta_{j}=\beta^{\star}$. (More precisely, we require $\beta^{\star}>\beta_{j}$ for all even $j \leq 2 m$ and $\beta^{\star}>u_{j}$ for all odd $j<2 m$.) Now let $u_{j}^{\star}=u_{j} \cup\left\{\beta_{j}\right\}$ for each $j \leq 2 m$. By construction, $\operatorname{tp}\left(u_{2 j}^{\star}, u_{2 j+1}^{\star}\right)=\operatorname{tp}\left(u_{2 j+2}^{\star}, u_{2 j+1}^{\star}\right)=t$ for all $j<m$. However, $\operatorname{tp}\left(u_{0}^{\star}, u_{2 m}^{\star}\right)=t$ may fail to hold: although $\operatorname{tp}\left(u_{0}, u_{2 m}\right)$ indeed equals $t^{\prime}$, it may not be the case that $u_{2 m}^{\star}\left(k_{0}-1\right)<u_{0}^{\star}(k-1)<u_{2 m}^{\star}\left(k_{0}\right)$, as tp $\left(u_{0}^{\star}, u_{2 m}^{\star}\right)=t$ would require.

We address this possibility by adding a $u_{2 m+1}^{\star}$ and $u_{2 m+2}^{\star}$ to our sequence and then 'rotating' it two steps. First, let $u_{2 m+2}^{\star}$ be any element of [Ord] ${ }^{k}$ such that

$\circ \operatorname{tp}\left(u_{0}^{\star}, u_{2 m+2}^{\star}\right)=t$,

$\circ$ if $k_{0}>0$, then $u_{2 m+2}^{\star}\left(k_{0}-1\right)<u_{2 m}^{\star}\left(k_{0}\right)$ (this is possible since $u_{0}^{\star}(k-2)<u_{2 m}^{\star}\left(k_{0}\right)$ and the latter is a limit ordinal) and

$\circ u_{2 m+2}^{\star}\left(k_{0}\right)>\beta^{\star}$. 
Now define $u_{2 m+1}^{\star}$ by letting $u_{2 m+1}^{\star} \uparrow(k-1)=u_{0}^{\star} \uparrow(k-1)$ and letting $u_{2 m+1}^{\star}(k-1)$ be any ordinal $\gamma>u_{0}^{\star}(k-2)$ such that

$$
\max \left\{u_{2 m+2}^{\star}\left(k_{0}-1\right), u_{2 m}^{\star}\left(k_{0}-1\right)\right\}<\gamma<u_{2 m}^{\star}\left(k_{0}\right)
$$

(and hence $\left.\gamma<u_{2 m+2}^{\star}\left(k_{0}\right)\right)$. Then

$$
\operatorname{tp}\left(u_{2 m+1}^{\star}, u_{2 m}^{\star}\right)=\operatorname{tp}\left(u_{2 m+1}^{\star}, u_{2 m+2}^{\star}\right)=\operatorname{tp}\left(u_{0}^{\star}, u_{2 m+2}^{\star}\right)=t .
$$

Therefore, if we define $\left\langle v_{j} \mid j \leq 2 m+2\right\rangle$ by setting $v_{0}=u_{2 m+1}^{\star}$, setting $v_{1}=u_{2 m+2}^{\star}$ and, for $2 \leq j<$ $2 m+2$, setting $v_{j}=u_{j-2}^{\star}$, we see that $\left\langle v_{j} \mid j \leq 2 m+2\right\rangle$ witnesses the conclusion of the theorem for $t$.

Readers may better appreciate the necessity of some argument like the foregoing after constructing a cycle as in Lemma 3.5 for the type 001011. For yet more on these matters, readers are referred to [19].

We turn now to the proof of our main lemma:

Proof of Lemma 3.4. By the weak compactness of $\kappa$, there exists a natural number $\ell$ and a $B \in[\kappa]^{\kappa}$ such that $\left|u_{\vec{\alpha}}\right|=\ell$ for all $\vec{\alpha} \in[B]^{n}$.

For any $\vec{\gamma}=\left\langle\gamma_{0}, \ldots, \gamma_{2 n-1}\right\rangle \in[B]^{2 n}$ and $I \in[2 n]^{n}$, let $\vec{\gamma}[I]=\left\langle\gamma_{i} \mid i \in I\right\rangle$ and

$$
U_{\vec{\gamma}}=\left\{\gamma_{0}, \ldots, \gamma_{2 n-1}\right\} \cup \bigcup_{I \in[2 n]^{n}} u_{\vec{\gamma}[I]} .
$$

Recall that $H(\omega)$ denotes the set of all hereditarily finite sets (in particular, $H(\omega)$ is itself a countable set). Define a colouring $c:[B]^{2 n} \rightarrow H(\omega)$ by letting

$$
c(\vec{\gamma})=\left\langle\operatorname{otp}\left(U_{\vec{\gamma}}\right),\left\langle\xi_{i} \mid i<2 n\right\rangle,\left\langle v_{I} \mid I \in[2 n]^{n}\right\rangle\right\rangle,
$$

where the latter sequences record the images of the $\gamma_{i}$ and $u_{\vec{\gamma}[I]}$, respectively, under the order isomorphism $h: U_{\vec{\gamma}} \rightarrow \operatorname{otp}\left(U_{\vec{\gamma}}\right)$; more precisely,

$\circ \xi_{i}=h\left(\gamma_{i}\right)$ for each $i<2 n$ and

○ $v_{I}=h\left[u_{\vec{\gamma}[I]}\right]$ for each $I \in[2 n]^{n}$.

Again by the weak compactness of $\kappa$, we may fix a set $A \in[B]^{\kappa}$ such that $c$ is constant on $[A]^{2 n}$.

We now define $u_{\vec{\alpha}}$ for $\vec{\alpha}$ in $[A]^{\leq n}$ by reverse induction on $|\vec{\alpha}|$. The base case $\left\{u_{\vec{\alpha}} \mid \vec{\alpha} \in[A]^{n}\right\}$ is given. In the induction step,

○ $0<m<n$,

$\circ u_{\vec{\beta}}$ is defined for all $\vec{\beta}$ in $[A]^{\leq n}$ of length greater than $m$ and

$\circ$ the $u_{\vec{\beta}}$ with $\vec{\beta}$ in $[A]^{m+1}$ are all of the same size.

Now fix $\vec{\alpha}$ in $[A]^{m}$.

Claim 3.6. The set $\left\{u_{\vec{\alpha}-\langle\beta\rangle} \mid \beta \in A \backslash(\max (\vec{\alpha})+1)\right\}$ forms a $\Delta$-system with a root $r$ such that $r<\beta$ and $r \sqsubseteq u_{\vec{\alpha}-\beta}$ for every $\beta \in A$ with $\beta>\max (\vec{\alpha})$.

Proof. First suppose that $m=n-1$. Consider any $\beta_{0}<\beta_{1}<\beta_{2}$ in $A \backslash(\max (\vec{\alpha})+1)$ together with any $\vec{\gamma}_{0}, \vec{\gamma}_{1}, \vec{\gamma}_{2}$ in $[A]^{2 n}$ such that

$\circ \vec{\alpha} \frown\left\langle\beta_{1}, \beta_{2}\right\rangle \sqsubseteq \vec{\gamma}_{0}$,

○ $\vec{\alpha} \frown\left\langle\beta_{0}, \beta_{2}\right\rangle \sqsubseteq \vec{\gamma}_{1}$,

$\circ \vec{\alpha} \frown\left\langle\beta_{0}, \beta_{1}\right\rangle \sqsubseteq \overrightarrow{\gamma_{2}}$. 
Let $\vec{\varepsilon}\left(\beta_{j}\right)=\vec{\alpha} \frown\left\langle\beta_{j}\right\rangle$ for $j<3$. Then $c\left(\vec{\gamma}_{0}\right)=c\left(\vec{\gamma}_{1}\right)=c\left(\vec{\gamma}_{2}\right)$ implies that

$$
\eta \in u_{\vec{\varepsilon}\left(\beta_{0}\right)} \cap u_{\vec{\varepsilon}\left(\beta_{1}\right)} \Leftrightarrow \eta \in u_{\vec{\varepsilon}\left(\beta_{0}\right)} \cap u_{\vec{\varepsilon}\left(\beta_{2}\right)} \Leftrightarrow \eta \in u_{\vec{\varepsilon}\left(\beta_{1}\right)} \cap u_{\vec{\varepsilon}\left(\beta_{2}\right)}
$$

for any $\eta$ in $\kappa$. It follows immediately that $\left\{u_{\vec{\alpha}}-\langle\beta\rangle \mid \beta \in A \backslash(\max (\vec{\alpha})+1)\right\}$ forms a $\Delta$-system. Let $r$ be its root. Routine pigeonhole arguments together with the homogeneity of $A$ then show that $r<\beta$ and $r \sqsubseteq u_{\vec{\alpha}-\langle\beta\rangle}$ for every $\beta \in A$ with $\beta>\max (\vec{\alpha})$.

Now suppose that $m<n-1$. We argue again essentially as above, beginning instead with a sequence $\beta_{0}<\beta_{1}<\beta_{2}<\delta_{0}<\cdots<\delta_{n-m-2}$ in $A \backslash(\max (\vec{\alpha})+1)$. Again take $\vec{\gamma}_{0} \sqsupseteq \vec{\alpha} \frown\left\langle\beta_{1}, \beta_{2}, \delta_{0}, \ldots, \delta_{n-m-2}\right\rangle$; similarly for $\vec{\gamma}_{1}$ and $\vec{\gamma}_{2}$. Let $\vec{\varepsilon}\left(\beta_{j}\right)=\vec{\alpha} \frown\left\langle\beta_{j}, \delta_{0}, \ldots, \delta_{n-m-2}\right\rangle$ for each $\beta_{j}$. Then again the $c$-homogeneity of $A$ implies that $\left\{u_{\vec{\varepsilon}(\beta)} \mid \beta \in\left(\alpha_{m-1}, \delta_{0}\right) \cap A\right\}$ forms a $\Delta$-system. Since $u_{\vec{\alpha}}\left\langle\langle\beta\rangle \sqsubseteq u_{\vec{\varepsilon}(\beta)}\right.$ for each such $\beta$, and since the sets $u_{\vec{\alpha} \frown\langle\beta\rangle}$ are all of the same size, $\left\{u_{\vec{\alpha} \frown\langle\beta\rangle} \mid \beta \in\left(\alpha_{m-1}, \delta_{0}\right) \cap A\right\}$ forms a $\Delta$ system as well. As $\delta_{0}$ was arbitrary, $\left\{u_{\vec{\alpha}}-\langle\beta\rangle \mid \beta \in A \backslash(\max (\vec{\alpha})+1)\right\}$ forms a $\Delta$-system; pigeonhole and homogeneity arguments, as above, secure the remainder of the claim.

Now let $u_{\vec{\alpha}}$ equal the root of the $\Delta$-system $\left\{u_{\vec{\alpha}-\langle\beta\rangle} \mid \beta \in A \backslash(\max (\vec{\alpha})+1)\right\}$. By Claim 3.6, the homogeneity of $A$ and a pigeonhole argument, we see that we have satisfied item 1 of Lemma 3.4. To see that we satisfy item $2 \mathrm{a}$ as well (and hence that our third inductive assumption was justified), observe that we may calculate $\left|u_{\vec{\alpha}}\right|$ from the constant value of $c \uparrow[A]^{2 n}$, which we denote by $\left\langle\ell^{*},\left\langle\xi_{i}^{*} \mid i<2 n\right\rangle,\left\langle v_{I}^{*} \mid I \in[2 n]^{n}\right\rangle\right\rangle$; the point is that this computation depends only on the size $m$ of $\vec{\alpha}$. To this end, let $\mathcal{J}_{m}=\left\{I \in[2 n]^{n} \mid I \cap m=m\right\}$. Consideration of $c(\vec{\gamma})$ for any $\vec{\gamma} \in[A]^{2 n}$ end-extending $\vec{\alpha}$ then shows that $\left|u_{\vec{\alpha}}\right|=\left|\bigcap_{I \in \mathcal{J}_{m}} v_{I}^{*}\right|$.

It remains to verify item 2 b. To this end, fix $m \leq n$ and an aligned pair of $m$-tuples $\vec{\alpha}, \vec{\beta} \subseteq A$. Suppose for the sake of contradiction that $u_{\vec{\alpha}}$ and $u_{\vec{\beta}}$ are not aligned, and fix $\eta \in u_{\vec{\alpha}} \cap u_{\vec{\beta}}$ such that $k_{0}=\left|u_{\vec{\alpha}} \cap \eta\right| \neq k_{1}=\left|u_{\vec{\beta}} \cap \eta\right|$. Let $t=\operatorname{tp}(\vec{\alpha}, \vec{\beta})$. Observe that if $\operatorname{tp}(\vec{\gamma}, \vec{\delta})=t$ for some $\vec{\gamma}, \vec{\delta} \in[A]^{m}$, then $u_{\vec{\gamma}}\left(k_{0}\right)=u_{\vec{\delta}}\left(k_{1}\right)$, by the homogeneity of $A$.

By Lemma 3.5 there exists an integer $\ell \geq 1$ and a sequence $\left\langle\vec{\gamma}_{j} \mid j \leq 2 \ell\right\rangle$ of elements of $[A]^{m}$ such that tp $\left(\vec{\gamma}_{0}, \vec{\gamma}_{2 \ell}\right)=t$ and tp $\left(\vec{\gamma}_{2 j}, \vec{\gamma}_{2 j+1}\right)=\operatorname{tp}\left(\vec{\gamma}_{2 j+2}, \vec{\gamma}_{2 j+1}\right)=t$ for all $j<\ell$. Let $\xi=u_{\vec{\gamma}_{0}}\left(k_{0}\right)$.

Claim 3.7. $u_{\vec{\gamma}_{2 j}}\left(k_{0}\right)=\xi$ for all $j<m$.

Proof. We proceed by induction on $j$. For $j=0$, the claim is trivial. Suppose that $0<j<m$ and $u_{\vec{\gamma}_{2 j-2}}\left(k_{0}\right)=\xi$. Then, since tp $\left(\vec{\gamma}_{2 j-2}, \vec{\gamma}_{2 j-1}\right)=t=\operatorname{tp}\left(\vec{\gamma}_{2 j}, \vec{\gamma}_{2 j-1}\right)$, we have $\xi=u_{\vec{\gamma}_{2 j-2}}\left(k_{0}\right)=$ $u_{\vec{\gamma}_{2 j-1}}\left(k_{1}\right)=u_{\vec{\gamma}_{2 j}}\left(k_{0}\right)$, as desired.

It follows from the claim that $u_{\vec{\gamma}_{2 m}}\left(k_{0}\right)=\xi$. However, since tp $\left(\vec{\gamma}_{0}, \vec{\gamma}_{2 m}\right)=t$, we also have $u_{\vec{\gamma}_{2 m}}\left(k_{1}\right)=$ $\xi$, contradicting the fact that $k_{0} \neq k_{1}$. This completes the proof of the lemma.

\section{Hechler forcing and $\lim ^{1} \mathrm{~A}$}

We begin this section with a few useful observations about Hechler forcing and finite-support iterations thereof. Recall that conditions in Hechler forcing are pairs $p=\left(s_{p}, f_{p}\right)$, where $s_{p} \in{ }^{<\omega} \omega$ and $f_{p} \in{ }^{\omega} \omega$. We will often call $s_{p}$ the stem of $p$. If $p$ and $q$ are conditions, then $q \leq p$ if and only if

○ $s_{q} \supseteq s_{p}$,

$\circ f_{q} \geq f_{p}$ and

○ $s_{q}(i)>f_{p}(i)$ for all $i \in \operatorname{dom}\left(s_{q}\right) \backslash \operatorname{dom}\left(s_{p}\right)$.

Observe that any two conditions with the same stem are compatible.

If $\mathbb{P}$ is a finite-support iteration of Hechler forcings, then the set of $p \in \mathbb{P}$ such that, for all $\alpha \in \operatorname{dom}(p)$, 
$\circ p \uparrow \alpha$ decides the value of $\dot{s}_{p(\alpha)}$ and

○ $\dot{f}_{p(\alpha)}$ is a nice $\mathbb{P}_{\alpha}$-name for an element of ${ }^{\omega} \omega$

is dense in $\mathbb{P}$. We will in general implicitly assume that all of our conditions come from this dense set.

The following situation will frequently arise:

Lemma 4.1. Suppose the following:

$\circ \mathbb{P}$ is a finite-support iteration of Hechler forcings.

$\circ \mathcal{A}$ is a finite collection of conditions in $\mathbb{P}$ such that $s_{p(\alpha)}=s_{q(\alpha)}$ for all $p, q \in \mathcal{A}$ and all $\alpha \in \operatorname{dom}(p) \cap \operatorname{dom}(q)$.

$\circ r \in \mathbb{P}$ and $r \leq p \uparrow(\max (\operatorname{dom}(r))+1)$ for every $p \in \mathcal{A}$.

Then the set $\mathcal{A} \cup\{r\}$ has a lower bound in $\mathbb{P}$.

Proof. We define such a lower bound $q$ in $\mathbb{P}$ as follows. First, let $\operatorname{dom}(q)=\operatorname{dom}(r) \cup \cup_{p \in \mathcal{A}} \operatorname{dom}(p)$. Next, let $q \uparrow(\max (\operatorname{dom}(r))+1)=r$. Finally, for all $\alpha \in \operatorname{dom}(q) \backslash(\max (\operatorname{dom}(r))+1)$, let $s_{q(\alpha)}=s_{p(\alpha)}$ for any $p \in \mathcal{A}$ with $\alpha \in \operatorname{dom}(p)$ and let $\dot{f}_{q(\alpha)}$ be a nice $\mathbb{P}_{\alpha}$-name for the pointwise maximum of the set $\left\{\dot{f}_{p(\alpha)} \mid p \in \mathcal{A}, \alpha \in \operatorname{dom}(p)\right\}$. It is now straightforward to verify that $q$ is a condition in $\mathbb{P}$ and as desired.

We now turn to proving a lemma about uniformising families of conditions in finite-support iterations of Hechler forcings. We first need the following consequence of weak compactness:

Lemma 4.2. Suppose that $\kappa$ is a weakly compact cardinal, $n<\omega$, and that $\left\langle X_{\vec{\alpha}} \mid \vec{\alpha} \in[\kappa] \leq n\right\rangle$ is a family of sets such that $\left|X_{\vec{\alpha}}\right|<\kappa$ for every $\vec{\alpha} \in[\kappa]^{\leq n}$. Suppose also that $c$ is a function with domain $[\kappa]^{n+1}$ such that for all $\vec{\beta} \in[\kappa]^{n+1}$, we have $c(\vec{\beta}) \in \prod_{i \leq n} X_{\vec{\beta} \backslash i}$. Then there is an unbounded set $A \subseteq \kappa$ such that for all $i \leq n$, all $\vec{\alpha} \in[A]^{i}$ and all $\vec{\gamma}, \vec{\delta} \in[A]^{n+1}$ end-extending $\vec{\alpha}$, we have $c(\vec{\gamma})(i)=c(\vec{\delta})(i)$.

Proof. The proof is a relatively straightforward modification of the classical ramification argument used to prove that $\kappa$ satisfies $\kappa \rightarrow(\kappa)_{\theta}^{n}$ for all $n<\omega$ and $\theta<\kappa$. We provide some details for completeness.

We proceed by induction on $n$. If $n=0$, then the result follows simply from the fact that $\kappa$ is regular and $\left|X_{\emptyset}\right|<\kappa$. Thus, suppose $n=m+1$ and we have established all instances of the result for $m$. By standard arguments (compare [17, Lemma 7.2]), using the strong inaccessibility of $\kappa$ and the fact that $\left|X_{\vec{\alpha}}\right|<\kappa$ for all $\vec{\alpha} \in[\kappa]^{\leq n}$, we can define a tree ordering $\left(\kappa,<_{c}\right)$ such that the following are true:

1. For all $\alpha<\beta<\kappa$, if $\alpha<_{c} \beta$, then $\alpha<\beta$.

2. For all $\alpha_{0}<_{c} \alpha_{1}<_{c} \cdots<_{c} \alpha_{m}<_{c} \gamma<_{c} \delta$, we have $c\left(\left\langle\alpha_{0}, \ldots, \alpha_{m}, \gamma\right\rangle\right)=c\left(\left\langle\alpha_{0}, \ldots, \alpha_{m}, \delta\right\rangle\right)$.

3. $\left(\kappa,<_{c}\right)$ is a $\kappa$-tree - that is, it has height $\kappa$ and every level has cardinality less than $\kappa$.

Since $\kappa$ is weakly compact and therefore has the tree property, we can fix an unbounded $A_{0} \subseteq \kappa$ such that $A_{0}$ is linearly ordered by $<_{c}$. Now define a function $d$ with domain $\left[A_{0}\right]^{n}$ as follows. For every $\vec{\alpha} \in\left[A_{0}\right]^{n}$, let $d(\vec{\alpha})=c(\vec{\alpha} \frown\langle\gamma\rangle) \uparrow n$ for some $\gamma \in A_{0} \backslash(\vec{\alpha}(m)+1)$. Note that by property 2 of $\left(\kappa,<_{c}\right)$ and the fact that $A_{0}$ is linearly ordered by $c$, the value of $d(\vec{\alpha})$ is independent of our choice of $\gamma$.

Apply the inductive hypothesis to find an unbounded $A \subseteq A_{0}$ such that for all $i \leq m$, all $\vec{\eta} \in[A]^{i}$ and all $\vec{\alpha}, \vec{\beta} \in[A]^{n}$ end-extending $\vec{\eta}$, we have $d(\vec{\alpha})(i)=d(\vec{\beta})(i)$. We claim that $A$ is as desired. To this end, fix $i \leq n, \vec{\alpha} \in[A]^{i}$ and $\vec{\gamma}, \vec{\delta} \in[A]^{n+1}$ end-extending $\vec{\alpha}$. If $i=n$, then the fact that $A$ is linearly ordered by $<_{c}$ implies that $c(\vec{\gamma})=c(\vec{\delta})$, so in particular, $c(\vec{\gamma})(i)=c(\vec{\delta})(i)$. If $i<n$, then by our definition of $d$ and our choice of $A$ we have

$$
c(\vec{\gamma})(i)=d(\vec{\gamma} \uparrow n)(i)=d(\vec{\delta} \uparrow n)(i)=c(\vec{\delta})(i),
$$

as required. 
Lemma 4.3. Let $n$ be a positive integer and let $\kappa$ be a weakly compact cardinal. Let $\left\langle p_{\vec{\alpha}} \mid \vec{\alpha} \in[\kappa]^{n}\right\rangle$ be a family of conditions in $\mathbb{P}$, a length- $\kappa$ finite-support iteration of Hechler forcings. Let $u_{\vec{\alpha}}=\operatorname{dom}\left(p_{\vec{\alpha}}\right)$. Then there are an unbounded set $A \subseteq \kappa$, a family $\left\langle u_{\vec{\alpha}} \mid \vec{\alpha} \in[A]^{<n}\right\rangle$, a natural number $\ell$ and a set of stems $\left\langle s_{i} \mid i<\ell\right\rangle$ such that the following are true:

1. $\left|u_{\vec{\alpha}}\right|=\ell$ for all $\vec{\alpha} \in[A]^{n}$, and if $\eta$ is the ith element of $u_{\vec{\alpha}}$, then $s_{p_{\vec{\alpha}}(\eta)}=s_{i}$.

2. A and $\left\langle u_{\vec{\alpha}} \mid \vec{\alpha} \in[A] \leq n\right\rangle$ satisfy the conclusions of Lemma 3.4.

3. $p_{\vec{\beta}} \uparrow u_{\vec{\alpha}}=p_{\vec{\gamma}} \uparrow u_{\vec{\alpha}}$ for all $\vec{\alpha} \in[A]^{<n}$ and $\vec{\beta}, \vec{\gamma} \in[A]^{n}$ such that $\vec{\alpha} \sqsubseteq \vec{\beta}$ and $\vec{\alpha} \sqsubseteq \vec{\gamma}$.

Proof. First, apply the weak compactness of $\kappa$ to the function on $[\kappa]^{n}$ defined by

$$
\vec{\alpha} \mapsto\left\langle s_{p_{\vec{\alpha}}(\eta)} \mid \eta \in \operatorname{dom}\left(p_{\vec{\alpha}}\right)\right\rangle
$$

to find an unbounded set $A_{0} \subseteq \kappa$, a natural number $\ell<\omega$ and a sequence $\left\langle s_{i} \mid i<\ell\right\rangle$ of stems such that for all $\vec{\alpha} \in\left[A_{0}\right]^{n}$, we have $\left|\operatorname{dom}\left(p_{\vec{\alpha}}\right)\right|=\ell$ and

$$
\left\langle s_{p_{\vec{\alpha}}(\eta)} \mid \eta \in \operatorname{dom}\left(p_{\vec{\alpha}}\right)\right\rangle=\left\langle s_{i} \mid i<\ell\right\rangle .
$$

Next, apply Lemma 3.4 to $\left\langle u_{\vec{\alpha}} \mid \vec{\alpha} \in\left[A_{0}\right]^{n}\right\rangle$ to find an unbounded set $A_{1} \subseteq A_{0}$ and sequence $\left\langle u_{\vec{\alpha}} \mid \vec{\alpha} \in\left[A_{1}\right]^{<n}\right\rangle$ as in the conclusion of that lemma.

For each $\vec{\alpha} \in\left[A_{1}\right]^{<n}$, let $X_{\vec{\alpha}}=\left\{p \in \mathbb{P} \mid \operatorname{dom}(p)=u_{\vec{\alpha}}\right\}$ and consider the function $c$ on $\left[A_{1}\right]^{n}$ defined as follows. For every $\vec{\beta} \in\left[A_{1}\right]^{n}$, define $c(\vec{\beta}) \in \prod_{i<n} X_{\vec{\beta}\lceil i}$ by letting $c(\vec{\beta})(i)=p_{\vec{\beta}} \uparrow u_{\vec{\beta}\lceil i}$ for all $i<n$.

Apply Lemma 4.2 to find an unbounded $A \subseteq A_{1}$ such that for all $i<n$, all $\vec{\alpha} \in[A]^{i}$ and all $\vec{\beta}, \vec{\gamma} \in[A]^{n}$ end-extending $\vec{\alpha}$, we have $c(\vec{\beta})(i)=c(\vec{\gamma})(i)-$ that is, $p_{\vec{\beta}} \uparrow u_{\vec{\alpha}}=p_{\vec{\gamma}} \uparrow u_{\vec{\alpha}}$. Then $A$, $\left\langle u_{\vec{\alpha}} \mid \vec{\alpha} \in[A] \leq n\right\rangle, \ell$ and $\left\langle s_{i} \mid i<\ell\right\rangle$ are as desired.

Remark 4.4. Suppose that $A,\left\langle u_{\vec{\alpha}} \mid \vec{\alpha} \in[A]^{\leq n}\right\rangle, \ell$ and $\left\langle s_{i} \mid i<\ell\right\rangle$ are as in the conclusion of Lemma 4.3. Given $\vec{\alpha} \in[A]^{<n}$, define $p_{\vec{\alpha}} \in \mathbb{P}$ by choosing any $\vec{\beta} \in[A]^{n}$ with $\vec{\alpha} \sqsubseteq \vec{\beta}$ and letting $p_{\vec{\alpha}}=p_{\vec{\beta}}\left\lceil u_{\vec{\alpha}}\right.$ (observe that this is independent of our choice of $\vec{\beta}$ ). The family $\left\langle p_{\vec{\alpha}} \mid \vec{\alpha} \in[A] \leq n\right\rangle$ then has the property that if $m \leq n$ and $\vec{\alpha}, \vec{\beta} \in[A]^{m}$ are aligned, then $p_{\vec{\alpha}}$ and $p_{\vec{\beta}}$ are compatible in $\mathbb{P}$.

We turn now to the first of our results on the vanishing of $\lim ^{n} \mathbf{A}$. In this case, the assumption of the existence of a weakly compact cardinal is certainly more than we need. For example, it is shown in [16] that $\lim ^{1} \mathbf{A}=0$ in any forcing extension by $\operatorname{Add}\left(\omega, \omega_{2}\right)$, the poset to add $\boldsymbol{\aleph}_{2}$-many Cohen reals. A similar argument shows that $\lim ^{1} \mathbf{A}=0$ in any forcing extension by a length- $\omega_{2}$ finite-support iteration of Hechler forcings, at least as long as the ground model satisfies $\mathrm{CH}$. This is to say that our main purpose in recording this theorem and proof is to foreground the essential logic of the more complicated arguments that will follow.

Theorem 4.5. Suppose that $\kappa$ is a weakly compact cardinal, and let $\mathbb{P}$ be a finite-support iteration of Hechler forcings of length $\kappa$. Then $V^{\mathbb{P}} \vDash{ }^{\prime} \lim ^{1} \boldsymbol{A}=0$ '.

Proof. For $\alpha<\kappa$, let $\dot{g}_{\alpha}$ be a $\mathbb{P}$-name for the $\alpha$ th Hechler real added by $\mathbb{P}$. In $V^{\mathbb{P}},\left\langle\dot{g}_{\alpha} \mid \alpha<\kappa\right\rangle$ is a $<^{*}$-increasing, cofinal sequence in ${ }^{\omega} \omega$, hence it suffices to show that in $V^{\mathbb{P}}$, every coherent family indexed by $\left\langle\dot{g}_{\alpha} \mid \alpha<\kappa\right\rangle$ is trivial. To this end, fix a condition $p_{0} \in \mathbb{P}$ and a sequence of $\mathbb{P}$-names $\dot{\Phi}=\left\langle\dot{\varphi}_{\alpha}: I\left(\dot{g}_{\alpha}\right) \rightarrow \mathbb{Z} \mid \alpha<\kappa\right\rangle$ forced by $p_{0}$ to be a coherent family. We will find a $q \leq p_{0}$ forcing $\dot{\Phi}$ to be trivial.

Let $A_{0}=\kappa \backslash\left(\max \left(\operatorname{dom}\left(p_{0}\right)\right)+1\right)$. Observe that for any $\alpha<\beta$ in $A_{0}$, there exists a $p \leq p_{0}$ such that $p \mathbb{P}_{\mathbb{P}}$ ' $g_{\alpha} \leq \dot{g}_{\beta}$ '. Namely, let $p=p_{0} \cup\left\{\left\langle\beta,\left(\emptyset, \dot{g}_{\alpha}\right)\right\rangle\right\}$. Hence for each $\alpha<\beta$ in $A_{0}$, since $p_{0}$ forces $\dot{\Phi}$ to be coherent, we may extend $p_{0}$ to a $q_{\alpha, \beta} \leq p_{0}$ such that for some $k_{\alpha, \beta}<\omega$,

$$
q_{\alpha, \beta} \mathbb{P}_{\mathbb{P}} ' g_{\alpha}^{\prime} \leq \dot{g}_{\beta} \text {, and } \dot{\varphi}_{\alpha}(k, m)=\dot{\varphi}_{\beta}(k, m) \text { for all } k \geq k_{\alpha, \beta} \text { and } m \leq \dot{g}_{\alpha}(k) \text { '. }
$$


Thin out $A_{0}$, if necessary, to an unbounded $A_{1}$ such that $k_{\alpha, \beta}$ equals some fixed $k^{*}$ for all $\langle\alpha, \beta\rangle \in\left[A_{1}\right]^{2}$. Now apply Lemma 4.3 to $\left\langle q_{\alpha, \beta} \mid\langle\alpha, \beta\rangle \in\left[A_{1}\right]^{2}\right\rangle$ to find an unbounded $A \subseteq A_{1}, u_{\emptyset},\left\langle u_{\langle\alpha\rangle} \mid \alpha \in A\right\rangle, \ell$ and $\left\langle s_{i} \mid i<\ell\right\rangle$ as in the statement of the lemma.

Define conditions $q_{\emptyset}$ and $\left\langle q_{\alpha} \mid \alpha \in A\right\rangle$ as follows. First, let $\alpha<\beta$ be elements of $A$ and let $q_{\emptyset}=q_{\alpha, \beta} \uparrow u_{\emptyset}$. By Lemma 4.3(3), this definition is independent of the choice of $\alpha$ and $\beta$. Next, for a fixed $\alpha \in A$, choose an arbitrary $\beta \in A$ with $\beta>\alpha$ and let $q_{\alpha}=q_{\alpha, \beta} \uparrow u_{\langle\alpha\rangle}$. Here again the choice of $\beta$ is immaterial.

Let $q=q_{\emptyset}$ and notice that, since $q_{\alpha, \beta} \leq p_{0}$ for all $\alpha<\beta$ in $A_{0}$, we also have $q \leq p_{0}$. We claim that $q$ forces $\dot{\Phi}$ to be trivial. Let $\dot{B}$ be a $\mathbb{P}$-name for the set of $\alpha \in A$ such that $q_{\alpha} \in \dot{G}$, where $\dot{G}$ is the canonical $\mathbb{P}$-name for the $\mathbb{P}$-generic filter.

Claim 4.6. $q \Vdash_{\mathbb{P}}$ ' $\dot{B}$ is unbounded in $\kappa$ '.

Proof. Let $r \leq q$ and $\eta<\kappa$ be arbitrary. It suffices to find an $\alpha \in(A \backslash \eta)$ such that $q_{\alpha}$ and $r$ are compatible. By construction, the sequence $\left\langle u_{\langle\alpha\rangle} \backslash u_{\emptyset} \mid \alpha \in A\right\rangle$ consists of pairwise disjoint sets, so $\left(u_{\langle\alpha\rangle} \backslash u_{\emptyset}\right) \cap \operatorname{dom}(r)=\emptyset$ for some $\alpha \in(A \backslash \eta)$. This implies that $q_{\alpha}$ is compatible with $r$, since $q_{\alpha} \uparrow u_{\emptyset}=q_{\emptyset}$ and $r \leq q_{\emptyset}$.

Claim 4.7. $q$ forces that, for all $\alpha<\beta$ in $\dot{B}$, there is a $\gamma \in(A \backslash \beta+1)$ such that $q_{\alpha, \gamma}$ and $q_{\beta, \gamma}$ are in $\dot{G}$.

Proof. Fix $r \leq q$ and $\alpha<\beta$ such that $r$ forces both $\alpha$ and $\beta$ to be in $\dot{B}$. By the definition of $\dot{B}$, we may assume that $r$ extends both $q_{\alpha}$ and $q_{\beta}$. It then suffices to find a $\gamma \in(A \backslash \beta+1)$ such that $r, q_{\alpha, \gamma}$ and $q_{\beta, \gamma}$ all have a common extension $s$. By construction, the families $\left\langle u_{\langle\alpha, \gamma\rangle} \backslash u_{\langle\alpha\rangle} \mid \gamma \in(A \backslash \beta+1)\right\rangle$ and $\left\langle u_{\langle\beta, \gamma\rangle} \backslash u_{\langle\beta\rangle} \mid \gamma \in(A \backslash \beta+1)\right\rangle$ each consist of pairwise disjoint sets. We can therefore find a $\gamma \in(A \backslash \beta+1)$ such that $\left(u_{\langle\alpha, \gamma\rangle} \backslash u_{\langle\alpha\rangle}\right) \cap \operatorname{dom}(r)=\emptyset$ and $\left(u_{\langle\beta, \gamma\rangle} \backslash u_{\langle\beta\rangle}\right) \cap \operatorname{dom}(r)=\emptyset$. By Lemma 3.4(2b), $u_{\langle\alpha, \gamma\rangle}$ and $u_{\langle\beta, \gamma\rangle}$ are aligned, and hence by Lemma 4.3(1), the stems in $q_{\alpha, \gamma}$ and $q_{\beta, \gamma}$ match whenever their domains intersect. Now apply Lemma 4.1 to $r$ and $\left\{q_{\alpha, \gamma}, q_{\beta, \gamma}\right\}$ to find a single condition $s$ extending all three conditions. This is the condition that we sought.

Claim 4.8. $q$ forces that, for all $\alpha<\beta$ in $\dot{B}$,

$$
\dot{\varphi}_{\alpha}(k, m)=\dot{\varphi}_{\beta}(k, m)
$$

for every $k \in \omega \backslash k^{*}$ and $m \leq \min \left(\left\{\dot{g}_{\alpha}(k), \dot{g}_{\beta}(k)\right\}\right)$.

Proof. Let $G$ be $\mathbb{P}$-generic with $q \in G$. Work in $V[G]$. Fix $\alpha<\beta$ in $B$. By Claim 4.7, we can find $\gamma \in(A \backslash \beta+1)$ such that $q_{\alpha, \gamma}$ and $q_{\beta, \gamma}$ are in $G$. This implies that

$\circ g_{\alpha} \leq g_{\gamma}$,

$\circ g_{\beta} \leq g_{\gamma}$ and

○ $\varphi_{\alpha}(k, m)=\varphi_{\gamma}(k, m)=\varphi_{\beta}(k, m)$ for all $k \geq k^{*}$ and $m \leq \min \left\{g_{\alpha}(k), g_{\beta}(k)\right\}$.

The claim follows.

Hence if $G$ is $\mathbb{P}$-generic with $q \in G$, then we may define a function $\psi: \omega \times \omega \rightarrow \omega$ trivialising $\Phi$ as follows. For all $(k, m) \in \omega \times \omega$, if there is an $\alpha \in B$ with $m \leq g_{\alpha}(k)$, then let $\psi(k, m)=\varphi_{\alpha}(k, m)$ for any such $\alpha$. Note that by Claim 4.8, if $k \geq k^{*}$, then the value of $\psi(k, m)$ is independent of our choice of $\alpha$. If there is no such $\alpha \in B$, let $\psi(k, m)=0$ (or any other integer). It is then straightforward to verify that $\psi$ does in fact trivialise $\Phi$, thus completing the proof of the theorem.

\section{Hechler forcing and $\lim ^{2} A$}

In this section we prove the case $n=2$ of our Main Theorem, which contains most of the key ideas of the general proof but is significantly less complex. We begin with some preliminary observations. 
Suppose that $\Phi=\left\langle\varphi_{f g}: I(f \wedge g) \rightarrow \mathbb{Z} \mid f, g \in{ }^{\omega} \omega\right\rangle$ is a 2-coherent family of functions. Recall that $\Phi$ determines a function $\partial^{1} \Phi$ taking each $(f, g, h) \in\left({ }^{\omega} \omega\right)^{3}$ to the function

$$
\varphi_{g h}-\varphi_{f h}+\varphi_{f g}: I(f \wedge g \wedge h) \rightarrow \mathbb{Z}
$$

For simplicity, in what follows we write $e$ for the difference function $\partial^{n} \Phi$ whenever $\Phi$ and $n$ are clear. Observe that when $\Phi$ is 2-coherent, every $e(f, g, h)$ is finitely supported; in this case, we write e $(f, g, h)$ for the restriction of $e(f, g, h)$ to its support.

Recall also that by the lemmas in Section 2.1, $\Phi$ is trivial if and only if there exists a $\leq^{*}$-cofinal family $F \subseteq{ }^{\omega} \omega$ and an alternating family

$$
\left\langle\psi_{f g}: I(f \wedge g) \rightarrow \mathbb{Z} \mid f, g \in F\right\rangle
$$

of finitely supported functions such that for all $f, g, h \in F$,

$$
e(f, g, h)=\left(\psi_{g h}-\psi_{f h}+\psi_{f g}\right) \uparrow I(f \wedge g \wedge h)
$$

When the functions in question are from an enumerated sequence $\left\langle f_{\alpha} \mid \alpha<\kappa\right\rangle$ in ${ }^{\omega} \omega$, we will often write $e(\alpha, \beta, \gamma)$ in place of $e\left(f_{\alpha}, f_{\beta}, f_{\gamma}\right)$, and $\varphi_{\alpha \beta}$ in place of $\varphi_{f_{\alpha} f_{\beta}}$, and so on. In such cases, due to the fact that all of our families of functions will be alternating, it will suffice to deal only with functions $\varphi_{\alpha \beta}$ for $\alpha<\beta$ and $e(\alpha, \beta, \gamma)$ for $\alpha<\beta<\gamma$, since these determine the rest of the functions in the relevant families. A similar statement will hold in the general case in Section 6.

Theorem 5.1. Suppose that $\kappa$ is a weakly compact cardinal, and let $\mathbb{P}$ be a finite-support iteration of Hechler forcing of length $\kappa$. Then $V^{\mathbb{P}} \vDash{ }^{\prime} \lim ^{2} A=0$ '.

Proof. As in the proof of Theorem 4.5, for all $\alpha<\kappa$ let $\dot{g}_{\alpha}$ be a nice $\mathbb{P}$-name for the Hechler real added at the $\alpha$ th stage of $\mathbb{P}$. Much as before, it will suffice to consider 2-coherent families in $V^{\mathbb{P}}$ indexed by pairs of functions from $\left\langle\dot{g}_{\alpha} \mid \alpha<\kappa\right\rangle$. Therefore fix a condition $p_{0} \in \mathbb{P}$ and a sequence of $\mathbb{P}$-names $\dot{\Phi}=\left\langle\dot{\varphi}_{\alpha \beta}: I\left(\dot{g}_{\alpha} \wedge \dot{g}_{\beta}\right) \rightarrow \mathbb{Z} \mid \alpha<\beta<\kappa\right\rangle$ forced by $p_{0}$ to be a 2-coherent family. We will find a $q \leq p_{0}$ which forces $\dot{\Phi}$ to be trivial.

Let $A_{0}=\kappa \backslash\left(\max \left(\operatorname{dom}\left(p_{0}\right)\right)+1\right)$. Again, much as before, for all $\alpha<\beta<\gamma$ in $A_{0}$, there exists a condition $q_{\alpha, \beta, \gamma} \leq p_{0}$ such that $q_{\alpha, \beta, \gamma} \mathbb{P}_{\mathbb{P}}$ ' $\dot{g}_{\alpha} \leq \dot{g}_{\beta} \leq \dot{g}_{\gamma}$ ' and $q_{\alpha, \beta, \gamma}$ decides the value of $\dot{\mathrm{e}}(\alpha, \beta, \gamma)$ to be equal to some $\epsilon_{\alpha, \beta, \gamma} \in V$. This condition exists because $\dot{e}(\alpha, \beta, \gamma)$ is forced by $p_{0}$ to be a finitely supported function. Thin out $A_{0}$ to an unbounded $A_{1}$ such that $\epsilon_{\alpha, \beta, \gamma}$ equals some fixed $\epsilon$ for all $\langle\alpha, \beta, \gamma\rangle \in\left[A_{1}\right]^{3}$.

Now apply Lemma 4.3 to $\left\langle q_{\alpha, \beta, \gamma} \mid\langle\alpha, \beta, \gamma\rangle \in\left[A_{1}\right]^{3}\right\rangle$ to find an unbounded $A \subseteq A_{1}$ together with sets $u_{\emptyset},\left\langle u_{\langle\alpha\rangle} \mid \alpha \in A\right\rangle$ and $\left\langle u_{\langle\alpha, \beta\rangle} \mid \alpha<\beta \in A\right\rangle$, a natural number $\ell$, and stems $\left\langle s_{i} \mid i<\ell\right\rangle$ as in the statement of the lemma. Define conditions $q_{\emptyset},\left\langle q_{\alpha} \mid \alpha \in A\right\rangle$ and $\left\langle q_{\alpha, \beta} \mid \alpha<\beta \in A\right\rangle$ as follows. First, for any $\alpha<\beta<\gamma$ in $A$, let $q_{\emptyset}=q_{\alpha, \beta, \gamma} \uparrow u_{\emptyset}$. Next, for each fixed $\alpha \in A$, choose $\beta<\gamma$ in $A$ with $\beta>\alpha$ and let $q_{\alpha}=q_{\alpha, \beta, \gamma} \uparrow u_{\langle\alpha\rangle}$. Finally, for fixed $\alpha<\beta$ in $A$, choose a $\gamma \in A$ with $\gamma>\beta$ and let $q_{\alpha, \beta}=q_{\alpha, \beta, \gamma} \uparrow u_{\langle\alpha, \beta\rangle}$. By Lemma 4.3(3), these definitions are independent of all of our choices.

Let $q=q_{\emptyset}$, and note that $q \leq p_{0}$. We claim that $q$ forces $\dot{\Phi}$ to be trivial. This we argue by first partitioning $A$ into two disjoint and unbounded subsets, $\Gamma_{1}$ and $\Gamma_{2}$. Let $\dot{B}$ be a $\mathbb{P}$-name for the set of $\alpha \in \Gamma_{1}$ such that $q_{\alpha} \in \dot{G}$, where $\dot{G}$ is the canonical name for the $\mathbb{P}$-generic filter. The proofs of the next two claims follow those of Claims 4.6 and 4.7 almost verbatim, so we omit them.

Claim 5.2. $q \Vdash_{\mathbb{P}}$ ' $\dot{B}$ is unbounded in $\kappa$ '.

Claim 5.3. $q$ forces that, for all $\alpha<\beta$ in $\dot{B}$, there is a $\gamma \in\left(\Gamma_{2} \backslash \beta+1\right)$ such that $q_{\alpha, \gamma}$ and $q_{\beta, \gamma}$ are in $\dot{G}$.

Claim 5.4. $q$ forces the following to hold in $V^{\mathbb{P}}$ : suppose that $\tau$ is in $[\dot{B}]^{3}$ and $\left\langle\alpha_{\sigma} \mid \sigma \in[\tau]^{2}\right\rangle$ is such that for all $\sigma \in[\tau]^{2}$ and all $\eta \in \sigma$, 
$\circ \alpha_{\sigma} \in \Gamma_{2} \backslash(\eta+1)$ and

$\circ q_{\eta, \alpha_{\sigma}} \in \dot{G}$.

Then there is an $\alpha_{\tau}$ in $A \backslash\left(\max \left\{\alpha_{\sigma} \mid \sigma \in[\tau]^{2}\right\}+1\right)$ such that for all $\sigma \in[\tau]^{2}$ and $\eta \in \sigma$, we have $q_{\eta, \alpha_{\sigma}, \alpha_{\tau}} \in \dot{G}$.

Proof. Fix $\tau \in\left[\Gamma_{1}\right]^{3},\left\{\alpha_{\sigma} \mid \sigma \in[\tau]^{2}\right\}$ and $r \leq q$ such that $r$ forces $\tau$ and $\left\{\alpha_{\sigma} \mid \sigma \in[\tau]^{2}\right\}$ to be as in the premise of the claim. Much as before, we may assume that $r$ extends $q_{\eta, \alpha_{\sigma}}$ for all $\sigma \in[\tau]^{2}$ and $\eta \in \sigma$. By construction, for all such $\sigma$ and $\eta$, the sequence $\left\langle u_{\left\langle\eta, \alpha_{\sigma}, \gamma\right\rangle} \backslash u_{\left\langle\eta, \alpha_{\sigma}\right\rangle} \mid \gamma \in\left(A \backslash \alpha_{\sigma}+1\right)\right\rangle$ consists of pairwise disjoint sets. Hence there exists an $\alpha_{\tau} \in A \backslash\left(\max \left\{\alpha_{\sigma} \mid \sigma \in[\tau]^{2}\right\}+1\right)$ such that

$$
\left(u_{\left\langle\eta, \alpha_{\sigma}, \alpha_{\tau}\right\rangle} \backslash u_{\langle\eta, \sigma\rangle}\right) \cap \operatorname{dom}(r)=\emptyset
$$

for all $\sigma \in[\tau]^{2}$ and $\eta \in \sigma$. Observe that for any $\sigma, \rho \in[\tau]^{2}, \eta \in \sigma$ and $\xi \in \rho$, the sets $\left\{\eta, \alpha_{\sigma}\right\}$ and $\left\{\xi, \alpha_{\rho}\right\}$ are aligned (it was to ensure this alignment that we partitioned $A$ into the disjoint sets $\Gamma_{1}$ and $\left.\Gamma_{2}\right)$. Therefore, by Lemma 3.4(2b), the sets $u_{\left\langle\eta, \alpha_{\sigma}, \alpha_{\tau}\right\rangle}$ and $u_{\left\langle\xi, \alpha_{\rho}, \alpha_{\tau}\right\rangle}$ are aligned, and hence by Lemma $4.3(1)$ the conditions $q_{\eta, \alpha_{\sigma}, \alpha_{\tau}}$ and $q_{\xi, \alpha_{\rho}, \alpha_{\tau}}$ are compatible, with identical stems wherever their domains intersect. Now apply Lemma 4.1 to $\left\langle q_{\eta, \alpha_{\sigma}, \alpha_{\tau}} \mid \sigma \in[\tau]^{2}, \eta \in \sigma\right\rangle$ and $r$ to find a single condition $s \in \mathbb{P}$ simultaneously extending all of these conditions. This $s$ forces $\alpha_{\tau}$ to be as desired.

We now turn more directly to the argument that $q$ forces $\dot{\Phi}$ to be trivial. Let $G$ be a $\mathbb{P}$-generic filter with $q \in G$; work in $V[G]$, and let $B$ and $\Phi$ be the realisations of $\dot{B}$ and $\dot{\Phi}$. Since $B$ is cofinal in $\kappa$, the family $\left\{g_{\eta} \mid \eta \in B\right\}$ is $\leq^{*}$-cofinal in ${ }^{\omega} \omega$. Therefore, it will suffice to find a family $\Psi=$ $\left\langle\psi_{\eta \xi}: I\left(g_{\eta} \wedge g_{\xi}\right) \rightarrow \mathbb{Z} \mid \eta<\xi \in B\right\rangle$ of finitely supported functions such that, for all $\eta_{0}<\eta_{1}<\eta_{2}$ in $B$,

$$
e\left(\eta_{0}, \eta_{1}, \eta_{2}\right)=\left(\psi_{\eta_{1} \eta_{2}}-\psi_{\eta_{0} \eta_{2}}+\psi_{\eta_{1} \eta_{2}}\right) \uparrow I\left(g_{\eta_{0}} \wedge g_{\eta_{1}} \wedge g_{\eta_{2}}\right)
$$

Using Claim 5.3, choose ordinals $\left\langle\alpha_{\sigma} \mid \sigma \in[B]^{2}\right\rangle$ such that $\alpha_{\sigma} \in\left(\Gamma_{2} \backslash \eta+1\right)$ and $q_{\eta, \alpha_{\sigma}} \in G$ for all $\sigma \in[B]^{2}$ and all $\eta \in \sigma$. Then, using Claim 5.4, choose ordinals $\left\langle\alpha_{\tau} \mid \tau \in[B]^{3}\right\rangle$ such that $\alpha_{\tau} \in\left(A \backslash \alpha_{\sigma}+1\right)$ and $q_{\eta, \alpha_{\sigma}, \alpha_{\tau}} \in G$ for all $\tau \in[B]^{3}$, all $\sigma \in[\tau]^{2}$ and all $\eta \in \sigma$. Together these choices ensure that $g_{\eta} \leq g_{\alpha_{\sigma}} \leq q_{\alpha_{\tau}}$ and $\mathrm{e}\left(\eta, \alpha_{\sigma}, \alpha_{\tau}\right)=\epsilon$ for all $\tau \in[B]^{3}$ and $\sigma \in[\tau]^{2}$ and $\eta \in \sigma$.

Now for arbitrary elements $\eta<\xi$ in $B$, let $\psi_{\eta \xi}=e\left(\eta, \xi, \alpha_{\{\eta, \xi\}}\right)$. Since $g_{\eta}$ and $g_{\xi}$ are both less than or equal to $g_{\alpha_{\{\eta, \xi\}}}$, the domain of $\psi_{\eta \xi}$ is in fact $I\left(g_{\eta} \wedge g_{\xi}\right)$, as desired. We claim that the family $\Psi$ so defined witnesses the triviality of $\Phi$.

To see this, let $\eta_{0}<\eta_{1}<\eta_{2}$ be arbitrary elements of $B$. To simplify notation, let $\alpha_{i}=\eta_{i}$ and $\alpha_{i j}=\alpha_{\left\{\eta_{i}, \eta_{j}\right\}}$ for $i<j<3$, and let $\alpha_{012}=\alpha_{\left\{\eta_{0}, \eta_{1}, \eta_{2}\right\}}$. We then have the following series of equations, each of which follows immediately from an expansion of expressions followed by a cancellation of like terms:

$$
\begin{array}{r}
e\left(\alpha_{1}, \alpha_{2}, \alpha_{012}\right)-e\left(\alpha_{0}, \alpha_{2}, \alpha_{012}\right)+e\left(\alpha_{0}, \alpha_{1}, \alpha_{012}\right)-e\left(\alpha_{0}, \alpha_{1}, \alpha_{2}\right)=0, \\
e\left(\alpha_{2}, \alpha_{12}, \alpha_{012}\right)-e\left(\alpha_{1}, \alpha_{12}, \alpha_{012}\right)+e\left(\alpha_{1}, \alpha_{2}, \alpha_{012}\right)-e\left(\alpha_{1}, \alpha_{2}, \alpha_{12}\right)=0, \\
e\left(\alpha_{2}, \alpha_{02}, \alpha_{012}\right)-e\left(\alpha_{0}, \alpha_{02}, \alpha_{012}\right)+e\left(\alpha_{0}, \alpha_{2}, \alpha_{012}\right)-e\left(\alpha_{0}, \alpha_{2}, \alpha_{02}\right)=0, \\
e\left(\alpha_{1}, \alpha_{01}, \alpha_{012}\right)-e\left(\alpha_{0}, \alpha_{01}, \alpha_{012}\right)+e\left(\alpha_{0}, \alpha_{1}, \alpha_{012}\right)-e\left(\alpha_{0}, \alpha_{1}, \alpha_{01}\right)=0 .
\end{array}
$$

The logic of the labelling is as follows: the operative indices in $\mathbf{a}_{012}$ are $0,1,2$ and 012 . The operative indices in $\mathbf{a}_{12}$ are 1,2,12 and 012; and similarly for $\mathbf{a}_{02}$ and $\mathbf{a}_{01}$. Here we have preferred readable equations to perfectly rigorous ones and have therefore omitted restriction notations; the essential observation about the domains of these functions is simply that $I\left(g_{\alpha_{0}} \wedge g_{\alpha_{1}} \wedge g_{\alpha_{2}}\right)$ is a subdomain of every one. This is because $g_{\eta} \leq g_{\alpha_{\sigma}} \leq g_{\alpha_{\tau}}$ for every $\tau \in[B]^{3}, \sigma \in[\tau]^{2}$ and $\eta \in \sigma$.

By construction, the restriction of each of the first two terms of $\mathbf{a}_{12}, \mathbf{a}_{02}$ and $\mathbf{a}_{01}$ to their support is equal to $e$. In consequence, these terms all cancel in the sum $\mathbf{a}_{12}-\mathbf{a}_{02}+\mathbf{a}_{01}-\mathbf{a}_{012}$, which reduces in 
turn, via cancellation of the third terms of $\mathbf{a}_{12}, \mathbf{a}_{02}$ and $\mathbf{a}_{01}$ with the like terms in $\mathbf{a}_{012}$, to

$$
e\left(\alpha_{0}, \alpha_{1}, \alpha_{2}\right)-e\left(\alpha_{1}, \alpha_{2}, \alpha_{12}\right)+e\left(\alpha_{0}, \alpha_{2}, \alpha_{02}\right)-e\left(\alpha_{0}, \alpha_{1}, \alpha_{01}\right)=0 .
$$

We may rewrite this equation as

$$
e\left(\alpha_{0}, \alpha_{1}, \alpha_{2}\right)=\left(\psi_{\alpha_{1} \alpha_{2}}-\psi_{\alpha_{0} \alpha_{2}}+\psi_{\alpha_{1} \alpha_{2}}\right) \uparrow I\left(g_{\alpha_{0}} \wedge g_{\alpha_{1}} \wedge g_{\alpha_{2}}\right) .
$$

This is the relation we desired; this concludes our proof.

\section{Hechler forcing and $\lim ^{n} \mathrm{~A}$}

In this section, we prove our Main Theorem. We prepare for the proof by first building up some technical machinery, frequently referring back to the proof of Theorem 5.1 for motivation. For the following sequence of definitions, let $\kappa$ be a fixed regular uncountable cardinal. Ultimately, of course, $\kappa$ will denote the weakly compact cardinal of our Main Theorem, but that hypothesis is irrelevant to Definitions and Claims 6.1 through 6.6.

Definition 6.1. For any nonempty $\tau$ in $[\kappa]^{<\omega}$, a subset-initial segment of $\tau$ is a sequence $\sigma_{1} \subseteq \cdots \subseteq$ $\sigma_{m} \subseteq \tau$ such that

$\circ \leq|\tau|$ and

○ $\left|\sigma_{i}\right|=i$ for all $i$ with $1 \leq i \leq m$.

We write $\vec{\sigma} \triangleleft \tau$ to indicate that $\vec{\sigma}$ is a subset-initial segment of $\tau$. If $m=|\tau|$, then we call $\vec{\sigma}=$ $\left\langle\sigma_{1}, \cdots, \sigma_{m}\right\rangle \triangleleft \tau$ a long string or long string for $\tau$.

Suppose now that $\left\langle g_{\alpha} \mid \alpha<\kappa\right\rangle$ is an injective sequence of elements of ${ }^{\omega} \omega$. Suppose that for each positive integer $n$, the family

$$
\Phi_{n}=\left\langle\varphi_{\vec{\alpha}}: I(\vec{\alpha}) \rightarrow \mathbb{Z} \mid \vec{\alpha} \in[\kappa]^{n}\right\rangle
$$

is $n$-coherent, where $I(\vec{\alpha})=I\left(\bigwedge_{i<n} g_{\alpha_{i}}\right)$ for each such $n$ and $\vec{\alpha} \in[\kappa]^{n}$. Let $\vec{\Phi}$ denote the family $\left\langle\varphi_{\vec{\alpha}} \mid \vec{\alpha} \in[\kappa]^{<\omega}, \vec{\alpha} \neq \emptyset\right\rangle$. Suppose also that $B \subseteq \kappa$ is unbounded and that to each nonempty $\tau \in[B]^{<\omega}$ we have assigned an ordinal $\alpha_{\tau}<\kappa$ in such a way that

$\circ$ if $\tau=\{\eta\}$, then $\alpha_{\tau}=\eta$, and

$\circ$ if $\rho \subsetneq \tau$, then $\alpha_{\rho}<\alpha_{\tau}$.

Given a nonempty $\tau \in[B]^{<\omega}$ and a subset-initial segment $\vec{\sigma} \triangleleft \tau$, write $\vec{\alpha}[\vec{\sigma}]$ to denote the sequence $\left\langle\alpha_{\sigma_{i}}|1 \leq i \leq| \vec{\sigma} \mid\right\rangle$; note that this sequence is increasing by assumption.

For $\vec{\alpha} \in[\kappa]^{<\omega}$ of length at least 2 , let

$$
e^{\vec{\Phi}}(\vec{\alpha})=\sum_{i<|\vec{\alpha}|}(-1)^{i} \varphi_{\vec{\alpha}^{i}}
$$

When the family $\vec{\Phi}$ is clear from context, it will be omitted from the superscript. Similarly, we will continue to notationally suppress the restriction of sums of functions to the intersection of their domains. Since each family $\Phi_{n}$ is $n$-coherent, the function $e(\vec{\alpha})$ is finitely supported for any $\vec{\alpha} \in[\kappa]^{<\omega}$ of length at least 2 . We write $\mathrm{e}(\vec{\alpha})$ for the restriction of $e(\vec{\alpha})$ to its support.

We now record a series of formal equalities. First, for all $\vec{\alpha} \in[\kappa]^{<\omega}$ of length at least 3 , let

$$
\mathrm{d} e(\vec{\alpha})=\sum_{\substack{i<|\vec{\alpha}| \\ \text { Press }}}(-1)^{i} e\left(\vec{\alpha}^{i}\right) .
$$


Observe that when the right-hand side of this equation is fully expanded, its terms will cancel; de is, after all, a composition of differentials. Hence $\operatorname{de}(\vec{\alpha})$ is well defined and equals 0 for all $\vec{\alpha} \in[\kappa]^{<\omega}$ of length at least 3 . If $L$ is some linear combination of the form

$$
\sum_{i<\ell} a_{i} e\left(\vec{\alpha}_{i}\right)
$$

with all $\vec{\alpha}_{i}$ of length at least 3 , then let

$$
\mathrm{d} L=\sum_{i<\ell} a_{i} \mathrm{~d} e\left(\vec{\alpha}_{i}\right) .
$$

Similarly, if $j$ is less than $\left|\vec{\alpha}_{i}\right|$ for all $i$, then let

$$
L^{j}=\sum_{i<\ell} a_{i} e\left(\vec{\alpha}_{i}^{j}\right) .
$$

Finally, here dropping the assumption that each $\vec{\alpha}_{i}$ has length at least 3 , for any $\vec{\beta} \in[\kappa]^{<\omega}$ with $\vec{\alpha}_{i}<\vec{\beta}$ for all $i<\ell$, let

$$
L * \vec{\beta}=\sum_{i<\ell} a_{i} e\left(\vec{\alpha}_{i}, \vec{\beta}\right),
$$

where $e\left(\vec{\alpha}_{i}, \vec{\beta}\right)=e\left(\vec{\alpha}_{i}-\vec{\beta}\right)$. If $\vec{\beta}=\langle\beta\rangle$, then we will abuse notation and write $L * \beta$ and $e\left(\vec{\alpha}_{i}, \beta\right)$ instead of $L *\langle\beta\rangle$ and $e\left(\vec{\alpha}_{i},\langle\beta\rangle\right)$. Finally, if $j$ is less than $\left|\vec{\alpha}_{i}\right|$ for all $i$, then let

$$
L^{j}=\sum_{i<\ell} a_{i} e\left(\vec{\alpha}_{i}^{j}\right) .
$$

For integers $n \geq 2$, we now recursively define interrelated

○ expressions $\mathcal{A}_{n}^{\vec{\Phi}}(\rho)$ parametrised by $\rho \in[B]^{n}$,

$\circ$ expressions $\mathcal{S}_{n}^{\vec{\Phi}}(\tau)$ and $\mathcal{C}_{n}^{\vec{\Phi}}(\tau)$ parametrised by $\tau \in[B]^{n+1}$ and

○ statements $\mathfrak{u}_{n}^{\vec{\Phi}}(\tau)$ parametrised by $\tau \in[B]^{n+1}$.

Again, when the family $\vec{\Phi}$ is clear from context it is omitted from superscripts. In fact, these expressions will depend also on the collection $\left\langle\alpha_{\tau} \mid \tau \in[B]^{<\omega}\right\rangle$ fixed earlier, but this dependence is always plain enough that we ignore it, notationally, entirely.

To begin, let

$$
\mathcal{A}_{2}(\rho)=e\left(\rho, \alpha_{\rho}\right)
$$

for each $\rho \in[B]^{2}$. Recall that we interpret elements of $[\kappa]^{<\omega}$ as finite increasing sequences, so, for example, if $\rho=\left\langle\beta_{0}, \beta_{1}\right\rangle$, then $e\left(\rho, \alpha_{\rho}\right)$ denotes $e\left(\left\langle\beta_{0}, \beta_{1}, \alpha_{\rho}\right\rangle\right)$. Next, given $n$ with $2 \leq n<\omega$ and $\tau \in[B]^{n+1}$, if $\mathcal{A}_{n}\left(\tau^{i}\right)$ has been defined for all $i \leq n$, let

$$
\begin{aligned}
& \mathcal{S}_{n}(\tau)=\mathrm{d} e\left(\tau, \alpha_{\tau}\right)-\sum_{i<n+1}(-1)^{i} \mathrm{~d}\left[\mathcal{A}_{n}\left(\tau^{i}\right) * \alpha_{\tau}\right] \\
& \mathcal{C}_{n}(\tau)=e(\tau)-\sum_{i<n+1}(-1)^{i} \mathcal{A}_{n}\left(\tau^{i}\right)
\end{aligned}
$$

and let $\mathfrak{u}_{n}(\tau)$ denote the conjunction of the following two statements:

- There exists an $\epsilon$ such that e $(\vec{\alpha}[\vec{\sigma}])=\epsilon$ for every long string $\vec{\sigma} \triangleleft \tau$.

○ For all nonempty $\rho, \sigma$ with $\rho \subsetneq \sigma \subseteq \tau$, we have $g_{\alpha_{\rho}} \leq g_{\alpha_{\sigma}}$. 
Lastly, let

$$
\mathcal{A}_{n+1}(\tau)=(-1)^{n+1} \mathcal{C}_{n}(\tau) * \alpha_{\tau}
$$

Let us pause to connect these definitions with the proof of Theorem 5.1. There $B$ was an unbounded subset of $\kappa$. For $\rho \in[B]^{2}$, we set $\psi_{\rho}$ equal to $\mathcal{A}_{2}(\rho)$. Then by deducing equation (11) for an arbitrary $\tau \in[B]^{3}$, we showed that this assignment of values was as desired. In the language just introduced, equation (11) translates to $\mathcal{C}_{2}(\tau)=0$. Similarly, the expression $\mathbf{a}_{12}-\mathbf{a}_{02}+\mathbf{a}_{01}-\mathbf{a}_{012}$ corresponds to $-\mathcal{S}_{2}(\tau)$. By the definition of $B$, the condition $\mathfrak{u}_{2}(\tau)$ holds for all $\tau \in[B]^{3}$; the relation $\mathcal{C}_{2}(\tau)=-\mathcal{S}_{2}(\tau)$ then followed immediately. The terms in $\mathcal{S}_{2}(\tau)$ are all of the form $\mathrm{de}(\vec{\beta})$, hence $\mathcal{S}_{2}(\tau)=0$. This, in essence, was the deduction that $\mathcal{C}_{2}(\tau)=0$.

The following two lemmas are easily proven by induction, so their proofs are left to the reader.

Lemma 6.2. For all $n$ with $2 \leq n<\omega$, all $\rho \in[B]^{n}$ and all $\tau \in[B]^{n+1}$, the expressions $\mathcal{A}_{n}(\rho), \mathcal{S}_{n}(\tau)$ and $\mathrm{C}_{n}(\tau)$ are all of the form

$$
\sum_{i<\ell} a_{i} e\left(\vec{\alpha}_{i}\right)
$$

where for all $i<\ell$ we have the following:

○ $a_{i} \in \mathbb{Z}$,

$\circ \vec{\alpha}_{i} \in[\kappa]^{n+1}$ and

$\circ \vec{\alpha}_{i}$ is of the form $\sigma_{0}-\left\langle\alpha_{\sigma_{j}} \mid 1 \leq j \leq\left(n+1-\left|\sigma_{0}\right|\right)\right\rangle$, where

$-0<\left|\sigma_{0}\right| \leq n+1$ and, if $\left|\sigma_{0}\right|<n+1$, then $1<\left|\sigma_{1}\right| \leq n+1$;

- if $\left|\sigma_{0}\right|<n+1$, then $\sigma_{0} \subseteq \sigma_{1}$;

- $\sigma_{j} \subsetneq \sigma_{j+1}$ for all $1 \leq j<\left(n+1-\left|\sigma_{0}\right|\right)$; and

- letting $k=\left(n+1-\left|\sigma_{0}\right|\right)$, we have $\sigma_{k} \subseteq \rho$ if we are considering $\mathcal{A}_{n}(\rho)$ and $\sigma_{k} \subseteq \tau$ if we are considering $\mathcal{S}_{n}(\tau)$ or $\mathcal{C}_{n}(\tau)$.

The point of this lemma is that although the expressions defining $\mathcal{A}_{n}(\rho), \mathcal{S}_{n}(\tau), \mathcal{C}_{n}(\tau)$ and $\mathfrak{u}_{n}(\tau)$ are constructed via a recursion referencing $\vec{\Phi}$, the values of $\mathcal{A}_{n}(\rho), \mathcal{S}_{n}(\tau)$ and $\mathcal{C}_{n}(\tau)$, and the truth values of $\mathfrak{u}_{n}(\tau)$ only ever depend on $\Phi_{n}$ and not on $\Phi_{m}$ for any $m \neq n$. Hence we may meaningfully use these expressions to argue as we do that $V^{\mathbb{P}} \vDash{ }^{\prime} \lim ^{n} \mathbf{A}=0$ ' while only referencing a single family $\Phi_{n}$ at a time. Similarly, since the values of $\mathcal{A}_{n}(\rho), \mathcal{S}_{n}(\tau)$ and $\mathcal{C}_{n}(\tau)$ depend on the ordinals $\left\langle\alpha_{\sigma} \mid \sigma \in[B]^{\leq n+1}\right\rangle$ but not on $\alpha_{\sigma}$ for $|\sigma|>n+1$, verifications via these expressions that $\lim ^{n} \mathbf{A}=0$ will never require that any ordinal $\alpha_{\sigma}$ of longer index $(|\sigma|>n+1)$ has yet been defined.

We can say more of $\mathcal{S}_{n}(\tau)$ than in the previous lemma:

Lemma 6.3. For all $n$ with $2 \leq n<\omega$ and all $\tau \in[B]^{n+1}$, the expression $\mathcal{S}_{n}(\tau)$ is of the form

$$
\sum_{i<\ell} b_{i} \mathrm{~d} e\left(\vec{\beta}_{i}\right),
$$

where $b_{i} \in \mathbb{Z}$ and $\vec{\beta}_{i} \in[\kappa]^{n+2}$ for all $i<\ell$. Since $\mathrm{d} e(\vec{\beta})=0$ for all $\vec{\beta} \in[\kappa]^{n+2}$, it follows that $\mathcal{S}_{n}(\tau)=0$.

The following is the main technical lemma regarding these formal expressions. Recall that we often interpret an element $\tau \in[\kappa]^{n+1}$ as an increasing sequence, so, for example, the expression $I(\tau)$ denotes $I\left(\bigwedge_{\alpha \in \tau} g_{\alpha}\right)$.

Lemma 6.4. Suppose that $2 \leq n<\omega, \tau \in[B]^{n+1}$ and $\mathfrak{u}_{n}(\tau)$ holds. Then

$$
\mathcal{S}_{n}(\tau)=(-1)^{n+1} \mathcal{C}_{n}(\tau)
$$

Proof. We proceed by induction on $n$, in fact establishing the following strengthening of the lemma, which we maintain as an inductive hypothesis: 
$\mathfrak{u}_{n}(\tau)$ induces a reduction of $\mathcal{S}_{n}(\tau)$ to $(-1)^{n+1} \mathcal{C}_{n}(\tau)$ via

(1) the cancellation of identical terms appearing with opposite sign in the expansion of $\mathcal{S}_{n}(\tau)$ and

(2) the cancellation of terms of the form $e(\vec{\alpha}[\vec{\sigma}])$ in which $\vec{\sigma} \triangleleft \tau$ is a long string, on the principle that, in the presence of $\mathfrak{u}_{n}(\tau)$, these terms are all equal when restricted to the intersection of their domains.

Such cancellations will be referred to as cancellations of type 1 or 2 , respectively.

These cancellations could conceivably alter the domain of $\mathcal{S}_{n}(\tau)$. We first show that they do not; we show in particular that when $\mathfrak{u}_{n}(\tau)$ holds, the domain of both $\mathcal{S}_{n}(\tau)$ and $\mathcal{C}_{n}(\tau)$ is $I(\tau)$. By Lemma 6.2, every term in each of $\mathcal{S}_{n}(\tau)$ and $\mathcal{C}_{n}(\tau)$ is an integer multiple of $e(\vec{\alpha})$, where $\vec{\alpha}$ is of the form $\sigma_{0}^{-}\left\langle\alpha_{\sigma_{1}}, \ldots, \alpha_{\sigma_{k}}\right\rangle$, where the sequence $\left\langle\sigma_{0}, \ldots, \sigma_{k}\right\rangle$ is as described therein. In particular, $\sigma_{0} \subseteq \sigma_{j} \subseteq \tau$ for all $j \leq k$. The condition $\mathfrak{u}_{n}(\tau)$ then ensures that $g_{\eta} \leq g_{\alpha_{\sigma_{j}}}$ for all $\eta \in \sigma_{0}$ and $j \leq k$. It follows that $\operatorname{dom}(e(\vec{\alpha}))=I\left(\sigma_{0}\right) \supseteq I(\tau)$ and hence that the domains of $\mathcal{S}_{n}(\tau)$ and $\mathcal{C}_{n}(\tau)$ both contain $I(\tau)$. Since both expressions explicitly include the term $e(\tau)$, whose domain is $I(\tau)$, the desired equalities hold.

Now observe that the expression $\mathcal{S}_{n}(\tau)$ can be rewritten as follows:

$$
\begin{aligned}
\mathcal{S}_{n}(\tau)= & \mathrm{d} e\left(\tau, \alpha_{\tau}\right)-\sum_{i<n+1}(-1)^{i} \mathrm{~d}\left[\mathcal{A}_{n}\left(\tau^{i}\right) * \alpha_{\tau}\right] \\
= & \mathrm{d} e\left(\tau, \alpha_{\tau}\right)-\sum_{i<n+1}(-1)^{i} \sum_{j<n+2}(-1)^{j}\left[\mathcal{A}_{n}\left(\tau^{i}\right) * \alpha_{\tau}\right]^{j} \\
= & \mathrm{d} e\left(\tau, \alpha_{\tau}\right)-\sum_{i<n+1}(-1)^{i} \sum_{j<n+1}(-1)^{j}\left[\mathcal{A}_{n}\left(\tau^{i}\right) * \alpha_{\tau}\right]^{j}-\sum_{i<n+1}(-1)^{n+i+1} \mathcal{A}_{n}\left(\tau^{i}\right) \\
= & \sum_{i<n+1}(-1)^{i} e\left(\tau^{i}, \alpha_{\tau}\right)-\sum_{i<n+1}(-1)^{i} \sum_{j<n+1}(-1)^{j}\left[\mathcal{A}_{n}\left(\tau^{i}\right) * \alpha_{\tau}\right]^{j} \\
& +(-1)^{n+1} e(\tau)-\sum_{i<n+1}(-1)^{n+i+1} \mathcal{A}_{n}\left(\tau^{i}\right) \\
= & \sum_{i<n+1}(-1)^{i} e\left(\tau^{i}, \alpha_{\tau}\right)-\sum_{i<n+1}(-1)^{i} \sum_{j<n+1}(-1)^{j}\left[\mathcal{A}_{n}\left(\tau^{i}\right) * \alpha_{\tau}\right]^{j} \\
& +(-1)^{n+1} \mathcal{C}_{n}(\tau) .
\end{aligned}
$$

If type 1 and 2 cancellations reduce the expression on the starred line to zero, then we will have shown $\mathcal{S}_{n}(\tau)=(-1)^{n+1} \mathcal{C}_{n}(\tau)$ while maintaining our inductive hypothesis. This is our goal. We begin with the base case of $n=2$. This amounts simply to a more careful translation of the end of the proof of Theorem 5.1 into our current terminology.

Since $\mathcal{A}_{2}\left(\tau^{i}\right) * \alpha_{\tau}=e\left(\tau^{i}, \alpha_{\tau^{i}}, \alpha_{\tau}\right)$ for any fixed $i<3$, and hence $\left[\mathcal{A}_{2}\left(\tau^{i}\right) * \alpha_{\tau}\right]^{2}=e\left(\tau^{i}, \alpha_{\tau}\right)$, the starred line in this case rearranges to

$$
\begin{array}{r}
\sum_{i<3}(-1)^{i} e\left(\tau^{i}, \alpha_{\tau}\right)-\sum_{i<3}(-1)^{i} e\left(\tau^{i}, \alpha_{\tau}\right)-\sum_{i<3}(-1)^{i} \sum_{j<2}(-1)^{j}\left[\mathcal{A}_{2}\left(\tau^{i}\right) * \alpha_{\tau}\right]^{j} \\
=\sum_{i<3}(-1)^{i+1} \sum_{j<2}(-1)^{j}\left[\mathcal{A}_{2}\left(\tau^{i}\right) * \alpha_{\tau}\right]^{j}
\end{array}
$$

The only cancellation here is of type 1 .

Fix $i<3$ and $j<2$ and observe that $\left[\mathcal{A}_{2}\left(\tau^{i}\right) * \alpha_{\tau}\right]^{j}$ is of the form $e\left(\eta, \alpha_{\tau^{i}}, \alpha_{\tau}\right)$, with $\eta \in \tau^{i}$. But then $\vec{\sigma}=\left\langle\{\eta\}, \tau^{i}, \tau\right\rangle$ is a long string for $\tau$ and $e\left(\eta, \alpha_{\tau^{i}}, \alpha_{\tau}\right)=e(\vec{\alpha}[\vec{\sigma}])$. Hence by $\mathfrak{u}_{2}(\tau)$ there is a fixed $\epsilon$ such that the restriction of each such term to the common domain $I(\tau)$ is equal to $\epsilon$. The starred 
line thus reduces further to

$$
\sum_{i<3}(-1)^{i+1} \sum_{j<2}(-1)^{j}\left[\mathcal{A}_{2}\left(\tau^{i}\right) * \alpha_{\tau}\right]^{j}=\sum_{i<3}(-1)^{i+1}(\epsilon-\epsilon)=0,
$$

as desired. The reduction is evidently by way of cancellations of type 2 . This completes the base case.

Now assume that $n>2$ and that the inductive hypothesis holds for $n-1$. We need two claims.

Claim 6.5. For all $i<n+1$,

$$
\sum_{j<n+1}(-1)^{j}\left[\mathcal{A}_{n}\left(\tau^{i}\right) * \alpha_{\tau}\right]^{j}=(-1)^{n} \mathcal{S}_{n-1}\left(\tau^{i}\right) * \alpha_{\tau} .
$$

Proof. Rewrite the sum $\sum_{j<n+1}(-1)^{j}\left[\mathcal{A}_{n}\left(\tau^{i}\right) * \alpha_{\tau}\right]^{j}$ in the following sequence of steps; each simply consists of an application of the definitions of the expressions under consideration:

$$
\begin{aligned}
& \sum_{j<n+1}(-1)^{j}\left[(-1)^{n} \mathcal{C}_{n-1}\left(\tau^{i}\right) * \alpha_{\tau^{i}}\right]^{j} * \alpha_{\tau} \\
& =(-1)^{n} \sum_{j<n+1}(-1)^{j}\left[\left[e\left(\tau^{i}\right)-\sum_{k<n}(-1)^{k} \mathcal{A}_{n-1}\left(\left(\tau^{i}\right)^{k}\right)\right] * \alpha_{\tau^{i}}\right]^{j} * \alpha_{\tau} \\
& =(-1)^{n}\left[\mathrm{~d} e\left(\tau^{i}, \alpha_{\tau^{i}}\right)-\sum_{k<n}(-1)^{k} \mathrm{~d}\left[\mathcal{A}_{n-1}\left(\left(\tau^{i}\right)^{k}\right) * \alpha_{\tau^{i}}\right]\right] * \alpha_{\tau} \\
& =(-1)^{n} \mathcal{S}_{n-1}\left(\tau^{i}\right) * \alpha_{\tau} .
\end{aligned}
$$

Claim 6.6. For all $i<n+1$, the condition $\mathfrak{u}_{n}(\tau)$ implies that

$$
(-1)^{n} \mathcal{S}_{n-1}\left(\tau^{i}\right) * \alpha_{\tau}=\mathcal{C}_{n-1}\left(\tau^{i}\right) * \alpha_{\tau}
$$

via cancellations of type 1 and 2 .

Proof. By the inductive hypothesis applied to $\mathcal{S}_{n-1}\left(\tau^{i}\right)$, we know that the terms appearing in $\mathcal{S}_{n-1}\left(\tau^{i}\right)$ but not in $(-1)^{n} \mathcal{C}_{n-1}\left(\tau^{i}\right)$ pair off in pairs either of type 1 , meaning that they consist of identical terms with opposite signs, or of type 2 , meaning that they are of the form $\left(e\left(\vec{\alpha}\left[\vec{\sigma}_{0}\right]\right),-e\left(\vec{\alpha}\left[\vec{\sigma}_{1}\right]\right)\right)$, where $\vec{\sigma}_{0}$ and $\vec{\sigma}_{1}$ are long strings for $\tau^{i}$.

If such a pair $(t,-t)$ is of type 1 , then $t * \alpha_{\tau}$ and $-t * \alpha_{\tau}$ also form a type 1 cancelling pair in $\mathcal{S}_{n-1}\left(\tau^{i}\right) * \alpha_{\tau}$. Notice also that if $\vec{\sigma}$ is a long string for $\tau^{i}$, then $\vec{\sigma}^{*}=\vec{\sigma} \frown\langle\tau\rangle$ is a long string for $\tau$ and $\vec{\alpha}\left[\vec{\sigma}^{*}\right]=\vec{\alpha}[\vec{\sigma}]^{-}\left\langle\alpha_{\tau}\right\rangle$. Therefore, if $\left(e\left(\vec{\alpha}\left[\vec{\sigma}_{0}\right]\right),-e\left(\vec{\alpha}\left[\vec{\sigma}_{1}\right]\right)\right)$ is a type 2 pair of terms from $\mathcal{S}_{n-1}\left(\tau^{i}\right)$, then $e\left(\vec{\alpha}\left[\vec{\sigma}_{0}\right]\right) * \alpha_{\tau}=e\left(\vec{\alpha}\left[\vec{\sigma}_{0}^{*}\right]\right)$ and $-e\left(\vec{\alpha}\left[\vec{\sigma}_{1}\right]\right) * \alpha_{\tau}=-e\left(\vec{\alpha}\left[\vec{\sigma}_{1}^{*}\right]\right)$. The condition $\mathfrak{u}_{n}(\tau)$ then implies that these terms are equal to some $\epsilon$ and $-\epsilon$, respectively, and thus cancel in $\mathcal{S}_{n-1}\left(\tau^{i}\right) * \alpha_{\tau}$ in a type 2 manner.

The terms of $\mathcal{S}_{n-1}\left(\tau^{i}\right) * \alpha_{\tau}$ that remain after these cancellations are precisely those of the form $t * \alpha_{\tau}$, where $t$ appears in $(-1)^{n} \mathcal{C}_{n-1}\left(\tau^{i}\right)$. Hence $\mathcal{S}_{n-1}\left(\tau^{i}\right) * \alpha_{\tau}=(-1)^{n} \mathcal{C}_{n-1}\left(\tau^{i}\right) * \alpha_{\tau}$. Hence $(-1)^{n} \mathcal{S}_{n-1}\left(\tau^{i}\right) *$ $\alpha_{\tau}=\mathcal{C}_{n-1}\left(\tau^{i}\right)$ via cancellations of type 1 and 2 . 
By Claims 6.5 and 6.6, the starred line now reduces to the following:

$$
\begin{aligned}
& \sum_{i<n+1}(-1)^{i} e\left(\tau^{i}, \alpha_{\tau}\right)-\sum_{i<n+1}(-1)^{i}\left[\mathcal{C}_{n-1}\left(\tau^{i}\right) * \alpha_{\tau}\right] \\
& =\sum_{i<n+1}(-1)^{i} e\left(\tau^{i}, \alpha_{\tau}\right)-\sum_{i<n+1}(-1)^{i}\left[e\left(\tau^{i}, \alpha_{\tau}\right)-\sum_{j<n}(-1)^{j} \mathcal{A}_{n-1}\left(\left(\tau^{i}\right)^{j}\right) * \alpha_{\tau}\right] \\
& =\sum_{i<n+1} \sum_{j<n}(-1)^{i+j} \mathcal{A}_{n-1}\left(\left(\tau^{i}\right)^{j}\right) * \alpha_{\tau} .
\end{aligned}
$$

Observe that the only cancellation here is of type 1 . Now the key observation is that to each $\left(\tau^{i}\right)^{j}$ with $i \leq j<n$ there corresponds a $\left(\tau^{i^{\prime}}\right)^{j^{\prime}}$ with $j^{\prime}<i^{\prime}<n+1$, and vice versa. Namely, when $i^{\prime}=j+1$ and $j^{\prime}=i$, then $\left(\tau^{i}\right)^{j}=\left(\tau^{i^{\prime}}\right)^{j^{\prime}}$. In the foregoing sum, these each associate to the signs $(-1)^{i+j}$ and $(-1)^{i+j+1}$, respectively. We therefore conclude by rewriting that sum as

$$
\sum_{i \leq j<n}(-1)^{i+j} \mathcal{A}_{n-1}\left(\left(\tau^{i}\right)^{j}\right) * \alpha_{\tau}+\sum_{j<i<n+1}(-1)^{i+j} \mathcal{A}_{n-1}\left(\left(\tau^{i}\right)^{j}\right) * \alpha_{\tau}=0,
$$

where again all cancellations are of type 1 . This completes the induction step. It therefore completes the proof of Lemma 6.4.

We are now ready to prove our main result, which we restate here for convenience.

Main Theorem. Let $\kappa \in V$ be a weakly compact cardinal, and let $\mathbb{P}$ denote a length- $\kappa$ finite-support iteration of Hechler forcings. Then

$$
V^{\mathbb{P}} \vDash ' \lim ^{n} \boldsymbol{A}=0 \text { for all } n>0 \text { '. }
$$

Proof. We will show that any $n$-coherent family of functions in $V^{\mathbb{P}}$ is trivial. Since we have already done so for $n=1$, we will assume in what follows that $n>1$. For all $\alpha<\kappa$, let $\dot{g}_{\alpha}$ be a nice $\mathbb{P}$-name for the Hechler real added at the $\alpha$ th stage of $\mathbb{P}$. As in the cases where $n=1$ and $n=2$, it will suffice to consider $n$-coherent families in $V^{\mathbb{P}}$ indexed by $n$-tuples of functions from $\left\langle\dot{g}_{\alpha} \mid \alpha<\kappa\right\rangle$. Therefore, fix a condition $p_{0} \in \mathbb{P}$ and a family of $\mathbb{P}$-names $\dot{\Phi}_{n}=\left\langle\dot{\varphi}_{\vec{\alpha}}: \dot{I}(\vec{\alpha}) \rightarrow \mathbb{Z} \mid \vec{\alpha} \in[\kappa]^{n}\right\rangle$ forced by $p_{0}$ to be an $n$-coherent family of functions. We will find a $q \leq p_{0}$ which forces $\dot{\Phi}_{n}$ to be trivial.

Let $A_{0}=\kappa \backslash\left(\max \left(\operatorname{dom}\left(p_{0}\right)\right)+1\right)$. Much as before, for all $\vec{\alpha} \in\left[A_{0}\right]^{n+1}$ there exists a condition $q_{\vec{\alpha}} \leq p_{0}$ such that $q_{\vec{\alpha}} \mathbb{P}_{\mathbb{P}}$ ' $\dot{g}_{\alpha_{0}} \leq \cdots \leq \dot{g}_{\alpha_{i}} \leq \cdots \leq \dot{g}_{\alpha_{n}}$ ' and $q_{\vec{\alpha}}$ decides the value of $\dot{e}(\vec{\alpha})$ to be equal to some $\epsilon_{\vec{\alpha}} \in V$. Thin out $A_{0}$ to an unbounded $A_{1}$ such that $\epsilon_{\vec{\alpha}}$ equals some fixed $\epsilon$ for all $\vec{\alpha} \in\left[A_{1}\right]^{n+1}$.

Now apply Lemma 4.3 to $\left\langle q_{\vec{\alpha}} \mid \vec{\alpha} \in\left[A_{1}\right]^{n+1}\right\rangle$ to find an unbounded $A \subseteq A_{1}$ together with sets $\left\langle u_{\vec{\alpha}} \mid \vec{\alpha} \in[A]^{\leq n}\right\rangle$, a natural number $\ell$ and stems $\left\langle s_{i}\right| i\langle\ell\rangle$ as in the statement of the lemma. Next, define conditions $\left\langle q_{\vec{\alpha}} \mid \vec{\alpha} \in[A]^{\leq n}\right\rangle$ as follows: for each $\vec{\alpha}$ in $[A]^{\leq n}$, let $\vec{\beta}$ be an element of $[A]^{n+1}$ such that $\vec{\alpha} \sqsubseteq \vec{\beta}$ and let $q_{\vec{\alpha}}=q_{\vec{\beta}} \uparrow u_{\vec{\alpha}}$. As usual, by Lemma 4.3(3), these definitions are independent of all of our choices of $(n+1)$-tuples $\vec{\beta}$. We will sometimes abuse notation and write, for example, $q_{\alpha}$ instead of $q\langle\alpha\rangle$.

Let $q=q_{\emptyset}$, and note that $q \leq p_{0}$. We claim that $q$ forces $\dot{\Phi}$ to be trivial. This we argue by first partitioning $A$ into $n+1$ disjoint and unbounded subsets $\left\langle\Gamma_{i} \mid 1 \leq i \leq n+1\right\rangle$. Let $\dot{B}$ be a $\mathbb{P}$-name for the set of $\alpha \in \Gamma_{1}$ such that $q_{\alpha} \in \dot{G}$, where $\dot{G}$ is the canonical name for the $\mathbb{P}$-generic filter. By exactly the same reasoning as in the proofs of Theorems 4.5 and 5.1,

$$
q \Vdash_{\mathbb{P}} \text { ' } \dot{B} \text { is unbounded in } \kappa \text { '. }
$$


Claim 6.7. Fix $\tau \in[\kappa]^{n+1}$. The condition $q$ forces the following to hold in $V^{\mathbb{P}}$ : suppose that $m$ and $\left\langle\alpha_{\sigma}\right| \sigma \in[\tau]^{<m}$ and $\left.\sigma \neq \emptyset\right\rangle$ are such that

$\circ 1<m \leq n+1$,

$\circ \alpha_{\{\eta\}}=\eta$ for all $\eta \in \tau$,

- $\alpha_{\rho}<\alpha_{\sigma}$ whenever $\rho$ is a proper subset of $\sigma$,

$\circ \alpha_{\sigma} \in \Gamma_{|\sigma|}$ for all nonempty $\sigma \in[\tau]^{<m}$ and

$\circ$ for any $1 \leq \ell<m$ and subset-initial segment $\vec{\sigma} \triangleleft \tau$ of length $\ell$, we have $q_{\vec{\alpha}[\vec{\sigma}]} \in \dot{G}$. In particular, $\eta \in \dot{B}$ for all $\eta \in \tau$.

Then there exists a sequence $\left\langle\alpha_{\sigma} \mid \sigma \in[\tau]^{m}\right\rangle$ of elements of $\Gamma_{m}$ which together with $\left\langle\alpha_{\sigma} \mid \sigma \in\left([\tau]^{<m} \backslash\{\emptyset\}\right)\right\rangle$ satisfies the following:

$\circ \alpha_{\rho}<\alpha_{\sigma}$ whenever $\rho$ is a proper subset of $\sigma$ and

$\circ$ for any subset-initial segment $\vec{\sigma} \triangleleft \tau$ of length $m$, we have $q_{\vec{\alpha}[\vec{\sigma}]} \in \dot{G}$.

Proof. Fix an $m$ and $\left\langle\alpha_{\sigma} \mid \sigma \in[\tau]^{<m} \backslash\{\emptyset\}\right\rangle$ and an $r \leq q$ such that $r$ forces $m$ and $\left\langle\alpha_{\sigma} \mid \sigma \in[\tau]^{<m} \backslash\{\emptyset\}\right\rangle$ to be as in the premise of the claim. In particular, $r$ forces that $q_{\vec{\alpha}[\vec{\sigma}]}$ is in $\dot{G}$ for every $\vec{\sigma} \triangleleft \tau$ as in the premise of the claim. As before, we may assume that $r$ extends all such $q_{\vec{\alpha}[\vec{\sigma}]}$. For each $\sigma \in[\tau]^{m}$ and $\vec{\sigma} \triangleleft \sigma$ of length $m-1$, observe that the sequence $\left\langle u_{\vec{\alpha}[\vec{\sigma}]-\gamma} \backslash u_{\vec{\alpha}[\vec{\sigma}]} \mid \gamma \in \Gamma_{m} \backslash(\max (\vec{\alpha}[\vec{\sigma}])+1)\right\rangle$ consists of pairwise disjoint sets. Therefore, we can

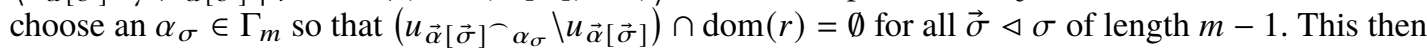
defines a family of pairwise-aligned sequences $\langle\vec{\alpha}[\vec{\sigma}]| \vec{\sigma} \triangleleft \tau$ is of length $m\rangle$, since for any $\alpha \in \Gamma_{k}$ in the intersection of two such $\vec{\alpha}[\vec{\sigma}]$ and $\vec{\alpha}[\vec{\rho}]$,

$$
|\vec{\alpha}[\vec{\sigma}] \cap \alpha|=|\vec{\alpha}[\vec{\rho}] \cap \alpha|=k .
$$

As before, this implies that the family $\left\langle q_{\vec{\alpha}[\vec{\sigma}]}\right| \vec{\sigma} \triangleleft \tau$ is of length $\left.m\right\rangle$ consists of pairwise compatible conditions, with identical stems wherever their domains intersect. Applying Lemma 4.1 to this collection together with $r$ then yields a lower bound $s \in \mathbb{P}$ for all of these conditions, thereby witnessing the conclusion of the claim.

As in the proof of Theorem 5.1, we will conclude our argument in $V[G]$, where $G$ is a $\mathbb{P}$-generic filter containing $q$. Let $B$ be the realisation of $\dot{B}$ and $\Phi_{n}$ the realisation of $\dot{\Phi}_{n}$ in $V[G]$. For each nonempty $\sigma \in[B]^{\leq n+1}$, we will specify an ordinal $\alpha_{\sigma}<\kappa$ in such a way that the collection $\left\langle\alpha_{\sigma} \mid \sigma \in[B]^{\leq n+1}\right\rangle$ satisfies the following:

$\circ \alpha_{\{\eta\}}=\eta$ for all $\eta \in B$,

$\circ \alpha_{\rho}<\alpha_{\sigma}$ whenever $\rho$ is a proper subset of $\sigma$,

$\circ \alpha_{\sigma} \in \Gamma_{|\sigma|}$ for all nonempty $\sigma \in[B]^{\leq n+1}$ and

$\circ$ for every $\tau \in[B]^{n+1}$ and every subset-initial segment $\vec{\sigma} \triangleleft \tau$, we have $q_{\vec{\alpha}[\vec{\sigma}]} \in G$.

The construction of $\left\langle\alpha_{\sigma} \mid \sigma \in[B]^{\leq n+1}\right\rangle$ proceeds via a straightforward recursion on $|\sigma|$, invoking Claim 6.7.

The $n$-coherent family $\Phi_{n}$ and collection $\left\langle\alpha_{\sigma} \mid \sigma \in[B]^{\leq n+1} \backslash\{\emptyset\}\right\rangle$ determine expressions $\mathcal{A}_{n}(\rho)$ for all $\rho \in[B]^{n}$ and expressions $\mathcal{S}_{n}(\tau)$ and $\mathcal{C}_{n}(\tau)$ and statements $\mathfrak{u}_{n}(\tau)$ for all $\tau \in[B]^{n+1}$. Note that our choices of $q, B$ and the sequence $\left\langle\alpha_{\sigma} \mid \sigma \in[B]^{\leq n+1}\right\rangle$ ensure that $\mathfrak{u}_{n}(\tau)$ holds for all $\tau \in[B]^{n+1}$.

For each $\rho \in[B]^{n}$, let $\psi_{\rho}=\mathcal{A}_{n}(\rho)$. It follows from the definition of $\mathcal{A}_{n}(\rho)$ and the fact that $\mathfrak{u}_{n}(\tau)$ holds for all $\tau \in[B]^{n+1}$ that $\mathcal{A}_{n}(\rho)$ is a finitely supported function with domain $I(\rho)$. To show that $\Phi_{n}$ is trivial, it will suffice to show that

$$
e(\tau)=\sum_{i<n+1}(-1)^{i} \psi_{\tau^{i}}
$$

for all $\tau \in[B]^{n+1}$. Fix such a $\tau$. Equation (12) is easily seen to be equivalent to the assertion that $\mathcal{C}_{n}(\tau)=0$. By Lemma 6.4 and the fact that $\mathfrak{u}_{n}(\tau)$ holds, $\mathcal{S}_{n}(\tau)=(-1)^{n+1} \mathcal{C}_{n}(\tau)$. By Lemma 6.3, 
$\mathcal{S}_{n}(\tau)=0$. In consequence, $\mathcal{C}_{n}(\tau)=0$. This shows that equation (12) holds for an arbitrary $\tau$, and hence that $\Phi_{n}$ is trivial.

\section{Conclusion}

Several natural questions follow immediately from our results in this paper. The first of these is whether the assumption of a weakly compact cardinal is necessary for the conclusion of the Main Theorem. As noted in Remark 1.2, this was answered in the negative by the authors together with Michael Hrušák in [5], where they showed that $\lim ^{n} \mathbf{A}=0$ for all $n>0$ after the addition of $\beth_{\omega}$-many Cohen reals. Closely related is the question of whether a large continuum is necessary for the conclusion of the Main Theorem.

Question 7.1. What is the minimum value of the continuum compatible with the statement ${ }^{\prime} \lim ^{n} \mathbf{A}=$ 0 for all $n>0$ '?

By the results of [22], that minimum value is at least $\boldsymbol{\aleph}_{2}$. By the aforementioned result in [5], $\boldsymbol{\aleph}_{\omega+1}$ is an upper bound for the answer to Question 7.1.

Our Main Theorem also lends the original, motivating questions a certain renewed charge:

Question 7.2. Is it consistent with the ZFC axioms (possibly modulo large cardinal assumptions) that strong homology is additive

$\circ$ on Polish spaces?

$\circ$ on locally compact metric spaces?

$\circ$ on metric spaces?

The extension $V^{\mathbb{P}}$ of the Main Theorem is, of course, a candidate model for an affirmative answer to any of these questions, and indeed, as noted in Remark 1.2, Bannister, Bergfalk and Moore recently showed in [1] that strong homology is additive on the class of locally compact Polish spaces in $V^{\mathbb{P}}$. A word is in order here about the machinery of strong homology, which consists first in an assignment of a system of approximations to a given topological space $X$ and second in an assignment of a homology group to $X$ by way of the system of homology groups of its approximations [21]. Compact metric spaces figure within this framework as particularly tractable: they admit sequential systems of approximations. Indeed, the index-set ${ }^{\omega} \omega$ so central to all our considerations arose as a countable product (induced by a countable topological sum) of just such a family of height- $\omega$ systems of approximations to $n$-dimensional Hawaiian earrings; the argument of [1] consists largely of correlating a broader class of ${ }^{\omega} \omega$-indexed inverse systems to strong homology computations for locally compact Polish spaces, and then showing that the present work's arguments apply to that broader class. In short, strong homology computations translate the ways that each of the bulleted classes in Question 7.2 relate to 'simpler' spaces to an associated family of index sets; the question is perhaps largely one of what sorts of combinatorics these index sets may or may not simultaneously support. Accordingly, the combinatorics of the interrelations among various partial orders may well play some role in its further solution (see, e.g., [31]). Through these interrelations, the vanishing of one system's higher derived limits may entail the vanishing of others'; [4, Theorem 5.1] is an example of such a result.

At some broader level, Question 7.2 is asking what sorts of continuities we may compatibly expect of homology functors on categories of topological spaces properly extending HCW - that is, properly including the category of spaces that are homotopy-equivalent to a $\mathrm{CW}$-complex. For the question 'On what class of spaces may a homology theory be both strong shape-invariant and additive?' is at heart a question about the interactions of the inverse and direct limits associated to the first prospective property and the second, respectively. As mentioned, closely related is the question of whether the strong homology groups of a space are the direct limits of the strong homology groups of its compact subspaces; a highly canonical extension of Steenrod homology exists on any class for which the answer is yes. This is the case in [1]; see its introduction and [22] and [21, Chapter 21.5] for further discussion. 
Lastly, one of this paper's referees asked what happens if we replace our iteration $\mathbb{P}$ of Hechler forcing with the standard length- $\kappa$ finite-support iteration $\mathbb{Q}$ for forcing Martin's axiom (MA) together with $2^{\aleph_{0}}=\kappa$. We conjecture that for all $n>0, \lim ^{n} \mathbf{A}=0$ will hold in $V^{\mathbb{Q}}$ as well, but our argument does not directly generalise to prove this, since it makes essential use of the fact that Hechler forcing is $\sigma$-centered (in particular, any finitely many conditions with the same stem have a common lower bound). This is evident in Lemmas 4.1 and 4.3(1), which are then used in the proof of Claim 6.7. There are straightforward generalisations of Lemmas 4.1 and 4.3 to arbitrary length- $\kappa$ finite-support iterations of $\sigma$-centered forcings of size $<\kappa$. (In this more general context, if $\mathbb{S}$ is a $\sigma$-centered poset and $p \in \mathbb{S}$, then $s_{p}$, the stem of $p$, is interpreted as the unique $j<\omega$ such that $p \in S_{j}$, where $\left\langle S_{j} \mid j<\omega\right\rangle$ is a fixed partition of the underlying set of $\mathbb{S}$ into centered subsets.) With this in mind, our proof adapts to show that if $\kappa$ is weakly compact and $\mathbb{Q}^{*}$ is the standard length- $\kappa$ finite-support iteration for forcing $\operatorname{MA}(\sigma$-centered $)+2^{\aleph_{0}}=\kappa$, then $\lim ^{n} \mathbf{A}=0$ for all $n>0$. The proof proceeds by considering $n$-coherent families indexed by $n$-tuples of the generic reals $\left\langle\dot{g}_{\alpha} \mid \alpha \in B\right\rangle$, where $B \in V$ is an unbounded subset of $\kappa$ such that for each $\alpha \in B$, the $\alpha$ th iterand of $\mathbb{Q}^{*}$ is a $\mathbb{Q}_{\alpha}^{*}$-name for Hechler forcing and $\dot{g}_{\alpha}$ is a name for the corresponding Hechler real. We therefore close with the question of the full form of Martin's axiom, and with thanks to the referee for asking it:

Question 7.3. Suppose that $\kappa$ is a weakly compact cardinal and that $\mathbb{Q}$ is the standard length- $\kappa$ finitesupport iteration for forcing MA $+2^{\aleph_{0}}=\kappa$. Is it the case that in $V^{\mathbb{Q}}, \lim ^{n} \mathbf{A}=0$ for all $n>0$ ?

Acknowledgments. This work began with a visit by the second author to UNAM Morelia. The authors would like to thank both VCU and UNAM for their support for this visit. More particularly, many of this paper's most fundamental impulses are due to Michael Hrušák and grew out of conversations with him both before and during the second author's visit; the authors would like to thank him very especially. The first author would like to thank Justin Moore and Jim West as well for much patient and formative instruction in the subject matter of this paper. The authors would also like to thank Stevo Todorcevic for encouraging them to reduce their large-cardinal assumption from a measurable to a weakly compact, and for his several suggestions for how to do so. Finally, they would like to thank both referees for their extremely thorough and thoughtful readings, comments and questions.

Conflict of Interest: None.

\section{References}

[1] N. Bannister, J. Bergfalk and J. T . Moore, 'On the additivity of strong homology for locally compact separable metric spaces', Preprint, 2020, arXiv:2008.13089.

[2] M. G. Barratt and J. Milnor, 'An example of anomalous singular homology', Proc. Amer. Math. Soc. 13 (1962), $293-297$.

[3] J. E. Baumgartner, 'Iterated forcing', in Surveys in Set Theory, London Mathematical Society Lecture Note Series, vol. 87 (Cambridge University Press, Cambridge, 1983), 1-59.

[4] J. Bergfalk, 'Strong homology, derived limits, and set theory', Fund. Math. 236(1) (2017), 71-82.

[5] J. Bergfalk, M. Hrušák and C. Lambie-Hanson, 'Simultaneously vanishing higher derived limits without large cardinals', Preprint, 2021, arXiv:2102.06699.

[6] J. Bergfalk, M. Hrušák and S. Shelah, 'Ramsey theory for highly connected monochromatic subgraphs', Acta Math. Hungar. 163(1) (2021), 309-322.

[7] J. Bergfalk and C. Lambie-Hanson, 'The cohomology of the ordinals I: Basic theory and consistency results', Preprint, 2019, arXiv: 1902.02736.

[8] D. Clausen and P. Scholze, Personal communication, July 2019.

[9] A. Dow, P. Simon and J. E. Vaughan, 'Strong homology and the proper forcing axiom', Proc. Amer. Math. Soc. 106(3) (1989), 821-828.

[10] K. Eda and K. Kawamura, 'The singular homology of the Hawaiian earring', J. Lond. Math. Soc. (2) 62(1) (2000), 305-310.

[11] P. C. Eklof and A. H. Mekler, Almost Free Modules, revised edn, North-Holland Mathematical Library, vol. 65 (NorthHolland Publishing Co., Amsterdam, 2002).

[12] I. Farah, 'A coherent family of partial functions on N', Proc. Amer. Math. Soc. 124(9) (1996), 2845-2852.

[13] R. Goblot, 'Sur les dérivés de certaines limites projectives. Applications aux modules', Bull. Sci. Math. (2) 94 (1970), 251-255.

[14] B. Günther, 'The Vietoris system in strong shape and strong homology', Fund. Math. 141(2) (1992), 147-168.

[15] C. U. Jensen, Les foncteurs dérivés de $\lim _{\longleftarrow}$ et leurs applications en théorie des modules, Lecture Notes in Mathematics, vol. 254 ( Springer-Verlag, Berlin-New York, 1972).

[16] S. Kamo, 'Almost coinciding families and gaps in $P(\omega)$ ', J. Math. Soc. Japan 45(2) (1993), 357-368. 
[17] A. Kanamori, The Higher Infinite, second edn, Springer Monographs in Mathematics (Springer-Verlag, Berlin, 2003). Large cardinals in set theory from their beginnings.

[18] K. Kunen, Set Theory, Studies in Logic and the Foundations of Mathematics, vol. 102 (North-Holland Publishing Co., Amsterdam-New York, 1980). An introduction to independence proofs.

[19] C. Lambie-Hanson, 'Disjoint type graphs with no short odd cycles', unpublished note, 2019, arXiv:2004.04189.

[20] J. T. Lisica, 'Strong bonding homology and cohomology', Topology Appl. 153(2-3) (2005), 394-447.

[21] S. Mardešić, Strong Shape and Homology, Springer Monographs in Mathematics ( Springer-Verlag, Berlin, 2000).

[22] S. Mardešić and A. V. Prasolov, 'Strong homology is not additive', Trans. Amer. Math. Soc. 307(2) (1988), $725-744$.

[23] J. Milnor, 'On axiomatic homology theory', Pacific J. Math. 12 (1962), 337-341.

[24] J. Milnor, 'On the Steenrod homology theory', in Novikov Conjectures, Index Theorems and Rigidity, Vol. 1 (Oberwolfach, 1993), London Mathematical Society Lecture Notes Series, vol. 226 (Cambridge University Press, Cambridge, 1995), 79-96.

[25] J. T. Moore, 'The proper forcing axiom', in Proceedings of the International Congress of Mathematicians. Volume II (Hindustan Book Agency, New Delhi, 2010), 3-29.

[26] B. L. Osofsky, 'The subscript of $\aleph_{n}$, projective dimension, and the vanishing of $\lim ^{(n)}$ ', Bull. Amer. Math. Soc. 80 (1974), $8-26$.

[27] A. V. Prasolov 'Non-additivity of strong homology', Topology Appl. 153(2-3) (2005), 493-527.

[28] P. Scholze, 'Lectures on condensed mathematics', unpublished lecture notes (2019). URL: https://www.math. uni-bonn.de/people/scholze/Condensed.pdf.

[29] J.-P. Serre, 'Faisceaux algébriques cohérents', Ann. of Math. (2) 61 (1955), 197-278.

[30] S. Shelah, 'Infinite abelian groups, Whitehead problem and some constructions', Israel J. Math. 18 (1974), $243-256$.

[31] S. Todorcevic, 'Directed sets and cofinal types', Trans. Amer. Math. Soc. 290(2) (1985), 711-723.

[32] S. Todorcevic, 'Reals and positive partition relations', in Logic, Methodology and Philosophy of Science, VII (Salzburg, 1983), Studies in Logic and the Foundations of Mathematics, vol. 114 (North-Holland, Amsterdam, 1986), 159- 169.

[33] S. Todorcevic, Partition Problems in Topology, Contemporary Mathematics, vol. 84 (American Mathematical Society, Providence, RI, 1989).

[34] S. Todorcevic 'The first derived limit and compactly $F_{\sigma}$ sets', J. Math. Soc. Japan 50(4) (1998), 831-836.

[35] B. Veličković and A. Vignati, In preparation.

[36] C. A. Weibel, An Introduction to Homological Algebra, Cambridge Studies in Advanced Mathematics, vol. 38 (Cambridge University Press, Cambridge, 1994). 